\title{
Masters of the Manipulator? A Comparative Genomics Study of Niveomyces gen. nov. and Two New Species of Mycoparasites Associated with Ophiocordyceps Camponoti-Floridani
}

Brittany M Lebert

University of Central Florida

João P.M. Araújo

UF: University of Florida

\section{Sara R Linehan}

University of Central Florida

Robin A Ohm

Utrecht University: Universiteit Utrecht

Andreas Brachmann

Ludwig-Maximilians-Universitat Munchen Fakultat fur Biologie

Charissa de Bekker ( $\nabla$ charissa.debekker@ucf.edu )

University of Central Florida https://orcid.org/0000-0002-7325-172X

\section{Research}

Keywords: Behavior manipulation, hyperparasites, mycoparasites, entomopathogens, comparative genomics, Hypocreales, Polycephalomyces

Posted Date: April 28th, 2021

DOI: https://doi.org/10.21203/rs.3.rs-449898/v1

License: (c) (i) This work is licensed under a Creative Commons Attribution 4.0 International License. Read Full License 


\section{Abstract}

Fungal hyperparasites can impact ecosystem composition and disease dynamics by modulating their parasite hosts' population size and transmission rate. Despite their perceived ecosystem impacts and potential to be applied in disease control efforts, hyperparasites are vastly understudied. In this integrative study we formally describe a new genus and two new mycoparasite species that infect the fungus Ophiocordyceps camponoti-floridani, which manipulates the behavior of Florida carpenter ants. Phylogenetic analyses demonstrate that both fungal hyperparasites are distinct species within the families Cordycipitaceae and Ophiocordycipitaceae. The unique morphology, ecology and phylogenetic placement of the Cordycipitaceae species supports its placement in a new genus, Niveomyces. Our field data alludes that both new species, Niveomyces ophiocordycipitis and Polycephalomyces oviedoensis, negatively impact $O$. camponoti-floridani survival and transmission. Moreover, their macromorphology, along with our field data, suggest that these hyperparasites live an exclusively mycoparasitic lifestyle and do not infect, nor decompose 0 . camponoti-floridani's ant host. To find genome signatures that would further confirm their mycoparasitism, we sequenced and annotated both species' genomes. We then compared the $N$. ophiocordycipitis and P. oviedoensis genomes to those of other mycoparasites, animal parasites, plant parasites, and saprophytes within the order Hypocreales and searched for previously reported mycoparasite genome signatures. However, our analyses indicate that these signatures are not greatly informative when expanding the number of genomes and lifestyles beyond those of previous studies, using the genome data that is currently available. This highlights the need for additional mycoparasite genomes within the order Hypocreales, as well as data on more closely related animal parasites. As such, this work contributes a starting point for more studies into fungal interactions between mycoparasites and entomopathogens, which have the potential to contribute important knowledge towards efforts to battle the global rise of plant and animal mycoses.

\section{Introduction}

Interspecies interactions are important drivers of evolution, biodiversity and ecosystem composition. Their significance is apparent throughout evolutionary history, where novel species interactions have led to major diversification events (Thompson 1999). Among the various forms of species-species interactions that exist, parasitism, where one species benefits from another at a cost to the benefactor's fitness, is highly common (Labaude et al. 2015). Considering the myriad of parasite species across all major taxa, including invertebrates, vertebrates, plants, and fungi, probably all free-living organisms are exploited by parasites. This highlights the importance of including parasites into studies that aim to understand the evolution, physiology, and behavior of organisms and their functioning within a larger ecological context (Windsor 1998). Indeed, previous studies have demonstrated both the direct and indirect effects of parasites on ecosystem composition through their ability to modulate their host's population size by increasing mortality or decreasing fecundity (Preston et al. 2016). In turn, these parasite-driven changes of host populations can significantly alter the entire community structure by impacting trophic cascades and other interspecies interactions (Frainer et al. 2018).

Since parasitism is such a successful lifestyle, which has evolved multiple times independently, most parasites are likely to also host parasites of their own. Such hyperparasites are expected to affect their host parasite's population size and disease dynamics, and exhibit additional indirect effects on ecosystem composition (Gleason et al. 2014). Indeed, it has been hypothesized that hyperparasites could control infectious diseases in certain species by regulating their parasites (Parratt and Laine 2016). This has resulted in the successful employment of a few thoroughly studied hyperparasites as biological control agents. For example, the CHV1 mycovirus is used as a biopesticide to control Cryphonectria parasitica, a parasitic fungus that causes blight in chestnut trees across the world (Feau et al. 2014). This demonstrates the high potential for hyperparasites to be employed in conservation efforts and against 
agricultural pests and other diseases, warranting detailed studies into tri-trophic hyperparasite-parasite-host relationships. Nevertheless, the impacts of hyperparasitism on the ecology and evolution of the parasites they affect, their hosts, and the parasite-parasite interactions involved, have not received much attention.

Fungal parasites are highly common and known to infect a wide range of hosts, including plants, insects, nematodes, and other fungi. Among them, a few fungal hyperparasites have also been reported. This includes Ampelomyces quisqualis, which infects members of the genus Erysiphaceae that cause Powdery Mildew Disease in several crop species (Park et al. 2010). The ability of $A$. quisqualis to reduce the spread of Powdery Mildew Disease has led to its use as a biopesticide (Park et al. 2010). Despite this, very few other hyperparasitic fungi have been studied in more detail beyond their species descriptions or reporting on their occurrence. This is especially true for the fungi that infect fungal entomopathogens, even though the fungal infection of insects is incredibly common and spans a wide biodiversity (Araújo and Hughes 2016). As such, the present study aims to add to the current very limited knowledge on fungal hyperparasites by describing one new genus, Niveomyces, and two new species, N. ophiocordycipitis and Polycephalomyces oviedoensis, that take part in tri-trophic interactions that involve another fungal parasite and its insect host. Both species parasitize the behavior-manipulating Zombie Ant Fungus that is commonly found in Central Florida; Ophiocordyceps camponoti-floridani (Araújo et al. 2018).

The genus Ophiocordyceps is composed of approximately 281 species, strictly associated with insects across 11 orders (Crous et al. 2004; Araújo and Hughes 2016). Among those, one particular group stands out for its intriguing and fascinating biology, the $O$. unilateralis clade sensu (Araújo et al. 2018). Ophiocordyceps unilateralis s.I. are known for infecting and manipulating the behavior of ants, mostly Camponotini, across the globe (Andersen et al. 2009; Evans et al. 2011). Typically, Ophiocordyceps-infected ants, such as the Florida Carpenter ant Camponotus floridanus, are manipulated to leave their nest and ascend vegetation, where they exhibit a fungus-adaptive "death-grip" behavior (Andersen et al. 2009; Araújo and Hughes 2019; Will et al. 2020). Once dead, Ophiocordyceps utilizes the ant's body as a food source to grow spore-producing structures needed for infection (Evans et al. 2011; Hughes et al. 2011; de Bekker et al. 2015; Araújo et al. 2018). Species within this group are known to produce two types of spores, conidia (asexual) and ascospores (sexual). Ascospores are produced and actively shot from the ascoma onto the forest floor where they potentially attach to ant workers that pass by while foraging for food. In contrast, the mucilaginous conidia are produced along the stalk, not actively shot and presumably transmitted to other ants by direct contact with the fungal structure (Araújo and Hughes 2017).

So far, the fungal hyperparasites of four other entomopathogenic Ophiocordyceps species from China and Thailand have been described (Wang et al. 2015b, a; Zhong et al. 2016; Xiao et al. 2018). However, none of these species are associated with a behavior manipulating Ophiocordyceps. In addition, hyperparasites growing on Ophiocordycepsmanipulated ants, such as Ophiocordyceps camponoti-rufipedis in Brazil (Andersen and Hughes 2012) and Ophiocordyceps paltothyrei in Ghana (Araújo et al. 2020), have been reported. Yet, their formal species descriptions and phylogenetic placement are currently lacking, as well as their evolutionary history. These cases, along with several other unpublished observations of hyperparasites on Ophiocordyceps across North and South America (personal communications), suggest that the tri-trophic interactions we discovered in Florida are not unique, but an example of a world-wide phenomenon. In spite of this, formal species descriptions and reports have remained limited, no genomes have currently been sequenced, and little research has been done on their biology and the effects that these hyperparasites have on Ophiocordyceps disease dynamics and transmission.

The integrative study presented here formally describes one new genus and two new hyperparasite species that affect Ophiocordyceps-ant host interactions in Central Florida, through both morphology, ecology and phylogenetic analysis. The species reside within the Cordycipitaceae and Ophiocordycipitaceae families of the order Hypocreales and were 
named Niveomyces ophiocordycipitis and Polycephalomyces oviedoensis, respectively. We also set out to learn more about their natural history by determining the timing of emergence and potential effects on 0 . camponoti-floridani. To further analyze their presumed mycoparasitic capabilities, we sequenced and annotated both hyperparasites' genomes. We searched for genome signatures that could confirm their hyperparasitic (i.e., mycoparasitic) lifestyles by comparing the two new genomes to the available genomes of other Hypocrealean fungi that represented mycoparasites, animal parasites, plant parasites, and saprophytes. Based on previous comparative genomics studies of fungal mycoparasites, we expected a typical mycoparasite genome to stand out by containing high numbers of GH18 chitinases, protease genes, and secondary metabolite clusters (Karlsson et al. 2017).

\section{Methods}

Field Study

As part of a larger longitudinal field study, we surveyed the occurrence of 0 . camponoti-floridani-infected ant cadavers from October 2018 to November 2019. Manipulated ant cadavers often cluster together in areas referred to as "graveyards" (Pontoppidan et al. 2009). As such, we chose nine of these graveyards to serve as our study sites. We set up three $9 \times 9$ meter graveyard plots in each of the following wilderness areas within Central Florida: Black Hammock

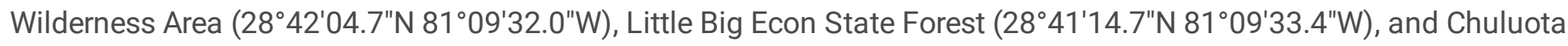
Wilderness Area $\left(28^{\circ} 37^{\prime} 22.8^{\prime \prime} \mathrm{N} 81^{\circ} 03^{\prime} 46.4^{\prime \prime W}\right)$. Permits were extended by the Seminole County's Leisure Services Department, Greenways and Natural Lands Division and the Florida Department of Agriculture and Consumer Service's Florida Forest Service. During a full year of observations, we tagged all infected ant cadavers within these nine plots, and recorded characteristics such as fruiting body growth and the presence or absence of a hyperparasite. During each plot visit, which occurred roughly every 1.5 months, we tagged new ant cadavers and recorded the growth and infection status of all previously tagged samples.

Sampling, DNA Extraction, Library Preparation \& Whole-Genome Sequencing

We collected hyperparasitized ant cadavers from the Black Hammock Wilderness Area in Oviedo, Florida. These specimens are readily recognized in the field by their characteristic cotton white hyphae and synnemata or dark perithecia that grow directly from the fungal hosts. Under a stereomicroscope, we removed hyperparasite tissues from the samples while taking careful consideration not to include 0 . camponoti-floridani tissue. We surface-sterilized the hyperparasite tissue in $70 \%$ ethanol and placed it into microcentrifuge tubes (Greiner, Monroe, NC, USA) along with two metal ball bearings (5/32" type 2B, grade 300, Wheels Manufacturing, Louisville, CO, USA). We snap-froze the fungal tissue in liquid nitrogen and used a 1600 MiniG tissue homogenizer (SPEX, Metuchen, NJ, USA) at 1300 RPM for 30 seconds to disrupt fungal cell walls. We then extracted DNA using a previously described phenol-chloroform extraction protocol (Will et al. 2020) and quantified it on a Qubit Fluorometer (Thermo Fisher, Waltham, MA, USA) with the Qubit dsDNA High Sensitivity Assay Kit (Thermo Fisher, Waltham, MA, USA). Subsequently, we prepared DNA libraries with the Nextera DNA Flex Library Preparation Kit (Illumina, San Diego, CA, USA) for sequencing on an Illumina MiSeq Sequencer to generate 2x250 bp paired-end reads with a 50x target coverage.

Genome Assembly \& Gene Prediction

Prior to assembling the two hyperparasite genomes, we filtered and trimmed our raw sequence data using the BBDuk plugin in Geneious Prime (20.2.3) with default parameters. Subsequently, to confirm the quality of these trimmed reads, we used fastQC (Andrews 2010). We then assembled the genomes de novo using the SPAdes assembly algorithm (Bankevich et al. 2012) and confirmed the quality of the assemblies through QUAST (Gurevich et al. 2013). As expected from samples taken directly from the field, the QUAST outputs showed slight bacterial contamination in 
the genomic data indicated by the presence of two distinct mean G-C\% peaks; one large peak comprising fungal reads (30-80\%) and a second, much smaller peak comprising bacterial contaminants $(0-30 \%)$. We removed the bacterial contaminants from both genomes by manually filtering out sequences with a mean $\mathrm{G}-\mathrm{C} \%$ that fell within the contaminant peak (i.e., <30\%). The effectiveness of this bacterial filtering was confirmed using MG-RAST (Keegan et al. 2016). We determined the completeness of the genomes after bacterial filtering with BUSCO (Seppey et al. 2019) by using the Hypocreales lineage (fungi_odb9), as well as CEGMA (Parra et al. 2007). Scaffolds shorter than 1000 bp were discarded. Gene prediction was performed using Augustus version 3.3 (Stanke and Morgenstern 2005). First, two separate sets of gene predictions were performed with the previously generated parameter set for Ophiocordyceps camponoti-floridani (Will et al. 2020) and the supplied parameter set of Fusarium graminearum. Next, these sets were combined into a final set by adding genes from the second set into the first set if those genes did not overlap with an existing gene in the first set. The annotated genomes are available through GenBank (NCBI) under the accession numbers: JADHZA000000000 (Polycephalomyces oviedoensis) and JAFEME000000000 (Niveomyces ophiocordycipitis). Moreover, the genome assembly, gene predictions and functional annotations can be analyzed interactively at http://fungalgenomics.science.uu.nl.

Functional Annotation

We functionally annotated the predicted proteins using PFAM (El-Gebali et al. 2019) and mapped them to their corresponding gene ontology (GO) terms. We predicted transmembrane domains using TMHMM 2.0 (Krogh et al. 2001) and signal peptides using SignalP-5.0 (Nielsen et al. 1997). Proteins with a secretory signal were considered small secreted proteins if they were shorter than 300 amino acids and did not contain a transmembrane domain (except in the first 40 amino acids). We used antiSMASH 5.0 (fungal version) to predict only well-defined and complete secondary metabolite clusters (Blin et al. 2019). We used BlastP, with an E-value cutoff of 1 e-10 to search the MEROPS database for proteases (Rawlings et al. 2018). Blast2Go was used to perform further blast searches on genes of interest (Gotz et al. 2008).

Comparative Genomics \& Orthologous Clustering

We compared the annotated hyperparasite genomes to other fungi with the following lifestyles: mycoparasites, animal parasites, plant parasites, and saprophytes. Mycoparasites included Tolypocladium ophioglossoides (Quandt et al. 2015), Trichoderma virens (Kubicek et al. 2011), Trichoderma atroviride (Kubicek et al. 2011), Trichoderma reesei (Martinez et al. 2008), Escovopsis weberi (de Man et al. 2016), and Clonostachys rosea (Karlsson et al. 2015). Animal parasites included Beauveria bassiana (Xiao et al. 2012), Metarhizium robertsii (Hu et al. 2014), Pochonia chlamydosporia (Larriba et al. 2014), and the hyperparasites' host Ophiocordyceps camponoti-floridani (Will et al. 2020). Plant parasites included Fusarium oxysporum (Asai et al. 2019) and Claviceps purpurea (Wingfield et al. 2018). Saprophytes included Stachybotrys chlorohalonata (Semeiks et al. 2014), Neurospora crassa (Baker et al. 2015), and Ophiostoma piceae (Haridas et al. 2013). These species are all members of the order Hypocreales, with the exception of $N$. crassa from Sordariales and 0 . piceae from Ophiostomatales. These two species were included due to the lack of available saprophyte genomes within Hypocreales. We functionally annotated the 15 genomes using the same methods as previously stated to ensure valid comparisons between the genomes of our two new species and the other fungal genomes. We identified orthologous gene clusters between the genomes using Orthofinder 2.4.0 (Emms and Kelly 2019) and visualized the summary output in a Venn diagram (http://bioinformatics.psb.ugent.be/webtools/Venn/). We used a Fisher Exact test with the Benjamini-Hochberg multiple testing correction (corrected $p$-value $<0.05$ ) to look for overrepresented functional annotation terms within these groups. 
Using our de novo assembled and annotated hyperparasites as protein Blast databases, we obtained sequences for ribosomal 18S (SSU), ribosomal 28S (LSU), translation elongation factor 1-alpha (Tef), and RNA Polymerase II Subunits (RPB1 and RPB2) for phylogenetic placement. The sequences that we obtained from the genomes were aligned in Geneious to a database of 445 species (Additional File 1) within the order Hypocreales. Each locus was individually aligned with MAFFT (Katoh and Standley 2013) and concatenated into a single combined dataset using Geneious $v$ 11.1.5. The concatenated files, along with a position (POS) file for each gene, were imported into CIPRES (Miller et al. 2012). The final alignment length was 4,747 bp: 1,024 bp for SSU, 949 bp for LSU, 960 bp for TEF, 688 bp for RPB1 and 1,126 bp for RPB2. Maximum likelihood analysis was performed with RAxML v 8.2.4 (Stamatakis 2014) on a concatenated dataset containing all five genes. The dataset consisted of 11 partitions, two for SSU and LSU and 9 for each codon position of the three protein coding genes: TEF, RPB1 and RPB2. The GTRGAMMA model of nucleotide substitution was employed during the generation of 1,000 bootstrap replicates. We visualized the tree using iTOL (Letunic and Bork 2019).

Morphology

To assess the macromorphology of the specimens, we took pictures using a Canon EOS 7D Mark II camera with a $35 \mathrm{~mm}$ lens. To investigate their micromorphological features, we stained fungal tissue on microscope slides with a drop of Lacto-Fuchsin stain (0.1g Acid Fuchsin Powder and $100 \mathrm{~mL}$ 85\% Lactic Acid). We visualized the slides using a Leica DMi8 inverted microscope, mounted with a Leica MC 170 HD camera (Leica Microsystems, Buffalo Grove, IL, USA). Type materials (holotypes and paratypes) are deposited at the New York Botanical Garden Fungarium (type numbers XXX and XXX for $P$. oviedoensis and $N$. ophiocordycipitis, respectively).

\section{Results}

\section{Phylogenetics}

Our phylogenetics results recovered the overall topology presented in previous studies (Sung et al. 2007; Quandt et al. 2015; Kepler et al. 2017; Araújo et al. 2018). Both Ophiocordycipitaceae and Cordycipitaceae families are well supported (BS = 90 and 100, respectively) as well as many of the internal nodes (Fig. 1). To determine the phylogenetic placement of the two hyperparasitic species that we discovered in Central Florida associated with $O$. camponoti-floridani, we generated a comprehensive phylogenetic tree of the order Hypocreales. Based on the phylogenetic results (Fig. 1), one of the two fungal hyperparasite species that infects 0 . camponoti-floridani is a distinct new species within the genus Polycephalomyces (Family: Ophiocordycipitaceae), which we have named $P$. oviedoensis (BS = 97). We sampled the genus Polycephalomyces broadly for our phylogenetics analysis and included a variety of species representing distinct ecologies such as animal and fungal parasites (Wang et al. 2015b, a; Zhong et al. 2016; Xiao et al. 2018). Our phylogeny indicates Polycephalomyces as the most basal lineage within Ophiocordyceps and supports $P$. oviedoensis sp. nov. as a distinct species (BS $=97$ ), closely related to a clade strictly associated with insects; P. cuboidea, P. paracuboidea and P. ryogamiensis (on Coleoptera) P. prolificus (on Hemiptera) (Fig. 1) (Kepler et al. 2013).

Figure 1: Phylogenetic placement of two new hyperparasitic fungal species of 0 . camponoti-floridani. A phylogenetic tree of the fungal order Hypocreales containing 445 representative species, including the two new species described in this study and their host 0 . camponoti-floridani. The new species reside within the families Cordycipitaceae and Ophiocordycipitaceae, which have been highlighted. Additionally, the clades for the two new species $P$. oviedoensis 
and $N$. ophiocordycipitis, as well as their host $O$. camponoti-floridani, are shown. The colors indicate reported lifestyles for each of the fungal species that that we found to be closely related to the species that are the focus of this study.

The second hyperparasite we found associated with 0 . camponoti-floridani is not only a new species, but a new genus that is sister of Blackwellomyces (Cordycipitaceae), a lineage that produces vivid orange to red stromata, strictly associated with larvae of Coleoptera and Lepidoptera (Mongkolsamrit et al. 2020). The BS support is not very strong (BS = 55), which could be explained by the limited available taxa for this part of the phylogenetic tree. However, the phylogenetic placement of this species (Fig. 1), in addition to its ecological and unique morphological features, strongly suggest that it represents a novel lineage within Cordycipitaceae. As such, a new genus is proposed herein, i.e., Niveomyces, to accommodate $N$. ophiocordycipitis sp nov. Furthermore, the fact that we found that one of the mycoparasites of 0 . camponoti-floridani to be an undescribed genus hints that hyperparasites of entomopathogens harbor a large reservoir of undocumented fungal diversity if we consider the diversity of its host worldwide (Araújo et al. 2018).

Taxonomy

Based on a combination of morphological, ecological and phylogenetic data, we introduce a new genus and two new species of mycoparasites. Polycephalomyces oviedoensis and Niveomyces ( $N$. ophiocordycipitis) were both collected parasitizing Ophiocordyceps camponoti-floridani, a ubiquitous parasite of the ant Camponotus floridanus in Florida, USA.

Niveomyces J.P.M. Araújo, B.M. Lebert \& C. de Bekker, gen. nov. - MycoBank MB839229

Etymology: Name reflects the "snowy" (Lat.: niveus) appearance of this fungus.

A genus of mycoparasitic fungi occupying the basal portion of the Cordycipitaceae family (order Hypocreales). Mycelium white to light yellow, often covering the host entirely. Vegetative hyphae septate and hyaline. Synnemata multiple, erect, not branched, tapering towards the apex, covered by hymenium-like layer of conidiogenous cells. Conidiogenous cells polyblastic, elongated, irregular, hyaline, cylindrical, with characteristic denticles that are crowded on the apical part and less frequent towards the base. Conidia globose to ovoid formed singly on the denticles. Sexual morph unknown.

Type species: Niveomyces ophiocordycipitis J. P. M. Araújo, B.M. Lebert \& C. de Bekker, sp, nov. - MycoBank MBXXXX; Fig. 2.

\section{Etymology}

Name reflects the association with the genus Ophiocordyceps.

\section{Typus}

USA, Florida, Seminole County, Oviedo, Little Big Econ State Forest, 2841'14.7"N 8109'33.4"W, from Ophiocordyceps camponoti-floridani on Camponotus floridanus, June 2017, de Bekker.

\section{Diagnosis}

Fungus growing on Ophiocordyceps camponoti-floridani, producing white mycelium covering the host almost entirely, producing multiple spike-like synnemata exhibiting abundant characteristic conidiogenous cells bearing multiple denticles, especially at the apical part, producing globose to ovoid conidia. 
Mycelium white to light yellow, growing abundantly on the host; Synnemata multiple, not branching, white, slender, erect, arising from the subiculum that covers the host almost entirely, narrowing towards the end, averaging $311.9 \mathrm{x}$ $65.2 \mu \mathrm{m}$; covered by a hymenium-like layer of abundant conidiogenous cells. Conidiogenous cells (12-) $17(-25) \mathrm{x}$ 1.5-2 $\mu \mathrm{m}$, polyblastic, cylindrical, hyaline, irregular, sometimes capitate, bearing crowded hyaline denticles on the apical part, sometimes descending sparsely along almost the entire cell. Conidia (3.7-) $4.5(-5.5) \times 1.5-2(-2.5) \mu \mathrm{m}$ formed singly on the denticles, solitary, ovoid to globose, one-celled, smooth-walled.

\section{Host}

Ophiocordyceps camponoti-floridani

\section{Distribution}

Florida, USA

Figure 2: Niveomyces ophiocordycipitis (A) N. ophiocordycipitis growing on Ophiocordyceps camponoti-floridani that parasitizes Camponotus floridanus, (B) Close-up of synnemata; (C) Close-up of the apical part of synnema; (D-E) Conidiogenous cells; (F) Hymenium-like layer of conidiogenous cells; (G) Close-up of conidiogenous cells with characteristic crown of denticles. Scale bars: (C) $200 \mu \mathrm{m}$; (D-F) $10 \mu \mathrm{m}$; (G) $5 \mu \mathrm{m}$.

Polycephalomyces oviedoensis J. P. M. Araújo, B. Lebert \& C. de Bekker, sp, nov. - MycoBank MBXXXX; Fig. 3.

\section{Etymology}

Named after the location this species was discovered, Oviedo - FL.

\section{Typus}

USA, Florida, Seminole County, Oviedo, Little Big Econ State Forest, 2841'14.7"N 8109'33.4"W, from Ophiocordyceps camponoti-floridani on Camponotus floridanus, June 2017, de Bekker.

\section{Diagnosis}

Fungus growing on Ophiocordyceps camponoti-floridani, producing black perithecia in multiple parts of the host, from synnemata and ascomata to fungal parts that emerge from the ant joints and sutures.

Mycelium cream to pale, growing sparsely; Perithecia $103.4 \times 58.9 \mu \mathrm{m}$, black, ovoid to pyriform, formed in multiples parts of the host. Ascus and ascospores not observed. Asexual morph not observed.

Host: Ophiocordyceps camponoti-floridani

Distribution: Florida, USA.

Figure 3 Polycephalomyces oviedoensis: (A) P. oviedoensis growing on Ophiocordyceps camponoti-floridani, which parasitizes Camponotus floridanus. (B) Perithecia under optical microscope; (C) Close-up of perithecia.

Hyperparasite Infections in Nature

To determine the prevalence of $P$. oviedoensis and $N$. ophiocordycipitis, we included a survey of the presence of hyperparasites in a larger field study that aimed to learn more about the natural history of O. camponoti-floridani. In this study, we tagged a total of 945 ant cadavers infected with 0 . camponoti-floridani in nine survey plots across three 
wilderness areas in Central Florida throughout the year. During this time, we found 62 ant cadavers that had their Ophiocordyceps visibly infected by a hyperparasite (Fig. 4A). Of these 62 hyperparasitized samples, 46 were infected by $P$. oviedoensis (i.e., had visible dark perithecia forming on top of 0 . camponoti-floridani mycelium) and only 16 were infected by $N$. ophiocordycipitis (i.e., had visible emergence of white cotton-like synnemata). Both hyperparasites were present in each of the three wilderness areas that we surveyed and often even co-occurred in the same plot. However, we did not observe any Ophiocordyceps-infected cadavers that were infected by both hyperparasites at the same time.

Figure 4: Hyperparasite infections of Ophiocordyceps camponoti-floridani in nature. (A) The number and percentage of $O$. camponoti-floridani samples found infected with either P. oviedoensis or $N$. ophiocordycipitis. (B) The month in which a newly hyperparasitized cadaver was first noticed. (C) The time between the appearance of a newly Ophiocordyceps-infected carpenter ant and its first noticeable infection with either $P$. oviedoensis or $N$. ophiocordycipitis. (D) The state of 0 . camponoti-floridani after infection by a hyperparasite as measured by the presence or absence of a fruiting body.

While new ant cadavers infected with 0 . camponoti-floridani seemed to appear year-round, we asked if the same was true for the two hyperparasite species $P$. oviedoensis and $N$. ophiocordycipitis or if their emergence was more seasonal. To answer this question, we noted the date on which we first observed visible hyperparasite growth on previously tagged 0 . camponoti-floridani samples. We omitted the 0 . camponoti-floridani samples from this analysis that were first discovered with a hyperparasite already present $(\mathrm{n}=21)$. Our data indicates that new $P$. oviedoensis infections appeared consistently throughout the year, with a possible peak in March/April (Fig. 4B). In contrast, we almost exclusively found new $N$. ophiocordycipitis infections in November, with only one sample found in July (Fig. 4B). Our small sample size did not allow us to perform reliable statistics on our data to confirm if certain months of the year produce significantly more infections of either hyperparasite species compared to others. However, our data does suggest that, for both hyperparasite species, there is a potential seasonal trend to their infection biology, which is more prominent for $N$. ophiocordycipitis than for P. oviedoensis.

In addition to recording when both hyperparasite species emerged throughout the year, we monitored how much time it took for either hyperparasite to emerge on a new 0 . camponoti-floridani-infected ant. Such data provides insights into the lifestyles of $P$. oviedoensis and $N$. ophiocordycipitis and aids to confirm their assumed hyperparasitism, and, thus, mycoparasitism. Appearance on living, thriving mycelium and stalks of $O$. camponoti-floridani would suggest a mycoparasitic lifestyle while appearance on older, dying tissue would indicate a saprophytic one, and consistent emergence together with Ophiocordyceps would suggest insect co-infection and, thus, a more entomopathogenic lifestyle. For this analysis, we again removed the samples that were first discovered with a hyperparasite already present, because of uncertainties with regards to timing of hyperparasite appearance. Among the samples that we monitored for hyperparasite emergence, both P. oviedoensis and N. ophiocordycipitis appeared as early as a week after a new 0 . camponoti-floridani-infected cadaver was found and as late as 9 months after (Fig. 4C). The later emergence of the hyperparasites, long after the ant's death, suggests that it is unlikely that either species would be an entomopathogen that coinfects the ant alongside 0 . camponoti-floridani. Additionally, the occurrence of both species early on, when $O$. camponoti-floridani is still actively growing its mycelium, stalk and ascoma, suggests that neither $P$. oviedoensis nor $N$. ophiocordycipitis would thrive on dead material, making a saprotrophic lifestyle very unlikely for both hyperparasitic species we studied. As such, these field observations support the notion that both fungi are indeed to be considered mycoparasitic hyperparasites.

Parasites negatively affect their host's fitness. Both hyperparasites we studied infect their 0 . camponoti-floridani host only after successful ant infection and manipulation, which are necessary for entomopathogen transmission.

Page $9 / 30$ 
However, before Ophiocordyceps can transmit, it also needs to successfully develop the stalk and ascoma that produce and release the infective spores (i.e., conidia and ascospores). As such, we documented the state of the $O$. camponoti-floridani stalk and ascoma after infection with P. oviedoensis and N. ophiocordycipitis to determine if these fungi would have a negative effect on transmission and, thus, their host's fitness. We found that about half of all hyperparasitized samples never produced an ascoma (Fig. 4D). Additionally, of those that did, only very few $P$. oviedoensis and $N$. ophiocordycipitis-infected samples were able to develop an ascoma after hyperparasite infection (10.9\% and 6.3\% respectively) (Fig. 4D). Without ascoma formation, O. camponoti-floridani cannot produce ascospores, which hampers transmission to other ants. Moreover, macromorphological investigation of the samples showed that $N$. ophiocordycipitis tissue often covers 0 . camponoti-floridani entirely. This presents another possible reduction of $O$. camponoti-floridani transmission, by creating a physical barrier to the dispersal of both conidia and ascospores. In addition, both hyperparasite infections appeared to kill 0 . camponoti-floridani since our attempts to extract RNA from hyperparasitized stalks failed (while we were able to extract RNA from uninfected stalks) (Additional File 2). This suggests that the lifespan of Ophiocordyceps, and, thus, the amount of time over which it can transmit, is often critically reduced. Taken together, these field observations support the hypothesis that both $P$. oviedoensis and $N$. ophiocordycipitis act as parasites and affect $O$. camponoti-floridani fitness by decreasing transmission.

\section{Comparative Genomics}

As part of their species descriptions, we sequenced and assembled the genomes of the novel fungal hyperparasites $P$. oviedoensis and N. ophiocordycipitis. Detailed statistics on their assembly, gene predictions, annotations and completeness can be found in Additional File 3. To describe these genomes in more detail and determine if they contain features that would further confirm the mycoparasitic lifestyles of $P$. oviedoensis and $N$. ophiocordycipitis as hyperparasites of 0 . camponoti-floridani, we compared them to 15 genomes. These genomes represented other fungal mycoparasites, animal parasites, plant parasites, and saprophytes, largely within the order Hypocreales.

We first used orthologous clustering to highlight the number of shared orthogroups between our two new hyperparasite species and the fungal genomes that represented the various fungal lifestyles in our comparison (Fig. 5A and B, Additional File 4). When compared to mycoparasites, P. oviedoensis uniquely shared 27 orthogroups, comprising 34 genes, with fungi representing this lifestyle, (Fig. 5A, Additional File 4, sheet a), while $N$. ophiocordycipitis uniquely shared 41 orthogroups, comprising 44 genes (Fig. 5B, Additional File 4, sheet b). However, both species shared more unique orthogroups with animal parasites (55 for $P$. oviedoensis and 190 for $N$. ophiocordycipitis) than mycoparasites (Fig. 5A and B, Additional File 4, sheet $\mathrm{C}$ and d). Both species uniquely shared the least amount of orthogroups with saprophytes (14 and 18 orthogroups, respectively). As such, our analysis indicates that the genomes of the two hyperparasites have more "parasite signatures" than "saprophytic signatures", which is in line with the expectations that we obtained from our field observations. However, we cannot state from this orthologous clustering analysis that either species genome suggests a specific mycoparasitic lifestyle. Analyzing the functional annotation terms of the shared orthologous genes between animal parasites and both hyperparasite species for potential enrichment showed that small secreted proteins (SSPs) were overrepresented. Similarly, we found an SSP enrichment when we analyzed the genes that the hyperparasites uniquely shared with both animal parasites and mycoparasites. However, no functional enrichments were found for the orthologous genes that the hyperparasites uniquely shared with only the mycoparasites in our comparison. In contrast, there was also an overrepresentation of SSPs among the 739 and 604 orthologs that were unique for each new hyperparasite species genomes (Fig. 5A and B, Additional File 4, sheet e and f). Only nine of these orthologs overlapped between them (Additional File 4, sheet g). While seven of these overlapping orthologs were proteins without a predicted function, we obtained a chitinase precursor blast result for one of these genes and another was predicted to code for an acyl-CoAacyltransferase, which is an enzyme involved in the biosynthesis of ergosterol (Zhang et al. 2020). 
Figure 5: Orthologous clustering. Venn diagrams representing the shared orthogroups between the genomes of $P$. oviedoensis (A) and $N$. ophiocordycipitis (B) and the genomes of 15 other fungi that we grouped into different fungal lifestyles based on their ability to parasitize animal, fungal, or plant hosts, or decompose dead organic material.

We subsequently used our orthologous clustering analysis to look for genes of interest that could reveal something about the general biology of the two new hyperparasite species. Many of the genes that were unique to either of the two species or uniquely shared with the myco- or animal parasites were annotated to be related to generic cell processes or were classified as hypothetical proteins with an unknown function. However, there were a few notable genes that stood out to because of their annotated function (Additional File 4). For example, N. ophiocordycipitis uniquely shared several genes with animal parasites that were annotated to be involved in antagonistic fungal interactions such as a hirsutellin A toxin, the mycotoxin cyclochlorotine (Schafhauser et al. 2016), and an efflux pump protein linked to antifungal resistance (Prasad and Rawal 2014). Comparably, the uniquely shared genes between $P$. oviedoensis and animal parasites included the antimicrobial peptide defensin (Wu et al. 2014) and an enterotoxin. The function of enterotoxins in insect-infecting fungi has been implicated to be involved in infection and even behavioral manipulation of the host (de Bekker et al. 2015; Will et al. 2020). However, N. ophiocordycipitis only contains one enterotoxin gene and $P$. oviedoensis only two, while many more enterotoxin genes are generally found in insect and nematode infecting fungi, with the number of enterotoxins being particularly high in Ophiocordyceps species (de Bekker et al. 2017). Nevertheless, beyond animal parasites, enterotoxins are generally not found at all in fungal genomes (de Bekker et al. 2017), which our study confirmed. None of the other genomes beyond the animal parasites and new hyperparasites species included in our study contained any annotated enterotoxin encoding genes.

A notable gene uniquely shared between $N$. ophiocordycipitis and other mycoparasites encoded a putative Heterokaryon Incompatibility (HET) Protein. These proteins allow fungi to sense the difference between their own growth and the growth of other fungi (Uehling et al. 2017), which could be a trait that is of particular importance for fungal mycoparasites. Indeed, P. oviedoensis and $N$. ophiocordycipitis both contain many HET genes (i.e., 18 genes and 37 genes, respectively) while their insect parasite host 0 . camponoti-floridani contains far fewer (i.e., only 6). However, when counting the number of HET genes in the other fungal genomes included in this study, they all appeared to have many more and no particular pattern related to lifestyle seemed to exist. Another gene that peaked our interest, because it was uniquely orthologous in $P$. oviedoensis and all mycoparasites in our comparison, was an extracellular membrane protein with an annotated CFEM domain that is thought to be involved in fungal pathogenesis (Kulkarni et al. 2003). However, both hyperparasites and their Ophiocordyceps host contain the same number of genes with this annotation (i.e., 10 genes), which indicates that perhaps besides this one ortholog, having genes with CFEM domains is not necessarily a feature indicative of mycoparasitism. When looking at the genes that were unique to $N$. ophiocordycipitis and P. oviedoensis (Fig. 5A and B, Additional File 3, sheet e and f), the majority ( 80\% and 70\%, respectively) did not have a known function. However, among the genes with a known function in N. ophiocordycipitis we found a ribotoxin alpha-sarcin, which has been found to exhibit strong antifungal properties (Citores et al. 2018) and might be involved in this species' mycoparasitic capabilities. Similarly, the unique $P$. oviedoensis genes included a putative gliotoxin gene and a Pathogenesis Related (PR-1-like) protein-encoding gene that has been linked to virulence in other fungal parasites (Prados-Rosales et al. 2012).

Our orthologous clustering analysis indeed suggested that the two new hyperparasites have a parasitic lifestyle. However, it did not specifically highlight a mycoparasitic lifestyle since we found lots of overlap with fungal parasites of animals as well. As such we continued our investigation into the hyperparasite genomes by asking if they contained the typical mycoparasite signatures that have previously been reported (Karlsson et al. 2017). Fungi are unique in their ability to produce a wide range of secondary metabolites, many of which have important bioactive properties (Brakhage 2013). Because these metabolites are not directly necessary for growth and development, their 
importance often lies in their contribution during interspecies interactions such as parasitism (Steenwyk et al. 2020). In addition, previous comparative genomic studies have suggested a link between high numbers of secondary metabolites and a mycoparasitic lifestyle (Karlsson et al. 2015). Although, these studies only compared mycoparasites to each other and did not include other fungal lifestyles. As such, the first signature we compared across all the genomes included in our study was the number and type of secondary metabolites clusters. We found that the average number of secondary metabolite clusters was 35.2 for mycoparasites, 41.8 for animal parasites, 30.0 for plant parasites, and 25.0 for saprophytes (Table 1). Overall, these cluster averages suggested that fungal parasites generally produced more secondary metabolites compared to saprophytes, with animal and mycoparasites producing the most, and that the number of clusters found in the hyperparasite genomes fit within this ballpark. However, when we further inspected the number of clusters for the individual species that represent the various fungal lifestyles, it became clear that there was a lot of variation between the species within each group (Table 1). As such, the number of secondary metabolite clusters did not seem to be an especially indicative genome signature to confirm a specifically mycoparasitic lifestyle as previously suggested. Nevertheless, investigating the potential secondary metabolites that these clusters produce, and their suggested function might still give us an indication about the lifestyles of the fungi that harbor them. The $P$. oviedoensis genome contained a total of 32 annotated secondary metabolite clusters that together comprised 200 genes (Table 1, Additional File 5, sheet 1). Of the 200 P. oviedoensis genes involved in secondary metabolism, $62.0 \%$ had orthologs in all other compared species while $7.0 \%$ were unique to this species. Out of the 32 clusters, only four of them matched to already known clusters within the antiSMASH database. One was $42.0 \%$ similar to a cluster that produces neosartorin, a compound known to have antibiotic properties in several Aspergillus species (Ola et al. 2014). The other three were all similar to clusters that are known to produce Cyclosporin C, a compound that has anti-fungal properties in several fungal species including Tolypocladium (Moussaïf et al. 1997). These bioactive secondary metabolites may play a role in infecting 0 . camponoti-floridanior protecting $P$. oviedoensis from attack by other microbes within their environment. We found that the $N$. ophiocordycipitis genome encoded a total of 38 secondary metabolite clusters that together included 347 genes (Table 1, Additional File 5, sheet 2). Of the 347 genes involved in secondary metabolism, 57.6\% had orthologs in all other compared species while $4.6 \%$ were unique to $N$. ophiocordycepitis. Out of the 38 clusters, six matched to already known clusters. One of these matched $100 \%$ to the epichloenin A secondary metabolite cluster. Epichloenin A has been found to play a role in fungus-plant interactions (Jia et al. 2019) and could perhaps play a role in $N$. ophiocordycipitis interactions with its fungal host $O$. camponoti-floridani as well. We found another cluster that matched for $83.0 \%$ with the chrysogine secondary metabolite pathway. Chrysogene is a yellow pigment known to protect fungal tissue from UV radiation and to have antibacterial properties in Penicillium (Viggiano et al. 2017). The $N$. ophiocordycipitis genome also contained a cluster that putatively produces an aspirochlorine-like mycotoxin (13.0\% match) (Wu et al. 2000). As such, it appears that both hyperparasites contain various secondary metabolite clusters that produce bioactive compounds, which are putatively involved in antagonistic microbe-microbe interactions. 
Table 1

Annotated secondary metabolite clusters. The total number of annotated secondary metabolite clusters in each genome compared in this study and the number of clusters per type of metabolite produced are presented in this table.

\begin{tabular}{|c|c|c|c|c|c|c|c|c|c|c|}
\hline Species & Total & \multicolumn{9}{|c|}{ Annotated secondary metabolite clusters per predicted metabolite class } \\
\hline PKS & & \multirow[t]{2}{*}{ NRPS } & \multirow[t]{2}{*}{ Terpene } & \multirow[t]{2}{*}{ Indole } & \multirow[t]{2}{*}{ Hybrid } & \multirow[t]{2}{*}{ Siderophore } & \multirow{2}{*}{$\begin{array}{l}\text { Beta } \\
\text { Lactone }\end{array}$} & \multirow{2}{*}{$\begin{array}{l}\text { Fungal } \\
\text { RiPP }\end{array}$} & & \\
\hline T1 & T3 & & & & & & & & & \\
\hline \multicolumn{11}{|c|}{ New Mycoparasite Species } \\
\hline $\begin{array}{l}\text { Polycephalomyces } \\
\text { oviedoensis }\end{array}$ & 32 & 8 & 0 & 15 & 5 & 1 & 3 & 0 & 0 & 0 \\
\hline $\begin{array}{l}\text { Niveomyces } \\
\text { ophiocordycipitis }\end{array}$ & 38 & 13 & 0 & 14 & 2 & 1 & 7 & 1 & 0 & 0 \\
\hline \multicolumn{11}{|l|}{ Mycoparasites } \\
\hline $\begin{array}{l}\text { Tolypocladium } \\
\text { ophioglossoides }\end{array}$ & 30 & 9 & 0 & 10 & 4 & 0 & 6 & 0 & 1 & 0 \\
\hline $\begin{array}{l}\text { Trichoderma } \\
\text { virens }\end{array}$ & 48 & 14 & 0 & 16 & 10 & 0 & 8 & 0 & 0 & 0 \\
\hline $\begin{array}{l}\text { Trichoderma } \\
\text { atroviride }\end{array}$ & 34 & 12 & 0 & 10 & 8 & 0 & 4 & 0 & 0 & 0 \\
\hline $\begin{array}{l}\text { Trichoderma } \\
\text { reesei }\end{array}$ & 27 & 9 & 0 & 6 & 8 & 0 & 4 & 0 & 0 & 0 \\
\hline Escovopsis weberi & 19 & 5 & 0 & 3 & 6 & 1 & 3 & 0 & 1 & 0 \\
\hline $\begin{array}{l}\text { Clonostachys } \\
\text { rosea }\end{array}$ & 53 & 25 & 0 & 14 & 6 & 1 & 6 & 0 & 1 & 0 \\
\hline \multicolumn{11}{|l|}{ Saprophytes } \\
\hline $\begin{array}{l}\text { Ophiostoma } \\
\text { piceae }\end{array}$ & 13 & 9 & 0 & 1 & 3 & 0 & 0 & 0 & 0 & 0 \\
\hline $\begin{array}{l}\text { Stachybotrys } \\
\text { chlorohalonata }\end{array}$ & 46 & 21 & 4 & 6 & 8 & 2 & 5 & 0 & 0 & 0 \\
\hline Neurospora crassa & 16 & 6 & 1 & 3 & 3 & 1 & 0 & 0 & 0 & 1 \\
\hline \multicolumn{11}{|l|}{ Animal Parasites } \\
\hline $\begin{array}{l}\text { Ophiocordyceps } \\
\text { camponoti- } \\
\text { floridani }\end{array}$ & 26 & 10 & 1 & 4 & 6 & 2 & 3 & 0 & 0 & 0 \\
\hline $\begin{array}{l}\text { Metarhizium } \\
\text { robertsii }\end{array}$ & 56 & 18 & 0 & 10 & 8 & 3 & 13 & 1 & 1 & 2 \\
\hline $\begin{array}{l}\text { Beauveria } \\
\text { bassiana }\end{array}$ & 39 & 9 & 0 & 15 & 8 & 0 & 7 & 0 & 0 & 0 \\
\hline $\begin{array}{l}\text { Pochonia } \\
\text { chlamydosporia }\end{array}$ & 46 & 13 & 0 & 12 & 11 & 0 & 8 & 0 & 1 & 1 \\
\hline Plant Parasites & & & & & & & & & & \\
\hline
\end{tabular}




\begin{tabular}{|lcccccccccc|}
\hline Species & Total & \multicolumn{7}{l|}{ Annotated secondary metabolite clusters per predicted metabolite class } \\
\hline $\begin{array}{l}\text { Fusarium } \\
\text { oxysporum }\end{array}$ & 40 & 10 & 1 & 10 & 11 & 2 & 4 & 0 & 2 & 0 \\
\hline $\begin{array}{l}\text { Claviceps } \\
\text { purpurea }\end{array}$ & 20 & 8 & 0 & 5 & 4 & 2 & 1 & 0 & 0 & 0 \\
\hline
\end{tabular}

Parasitic fungi secrete many proteases during infection (Monod et al. 2002). Indeed, mycoparasitic Trichoderma species appear to upregulate their proteases during contact with their host and have a noticeable percentage of protease encoding genes in their genomes (Druzhinina et al. 2012). Therefore, it has been suggested that the presence of a high number of protease genes in the genome would be characteristic of mycoparasitic fungi (Karlsson et al. 2017). When counting the number of putative protease encoding genes for our new hyperparasite species, we found that $P$. oviedoensis had a total of 266 (i.e., $3.2 \%$ of all genes annotated) and $N$. ophiocordycipitis had a total of 319 (i.e., 3.6\%) (Table 2, Additional File 6). These numbers place both new species within the expected percentages for mycoparasites and animal parasites, which respectively contained $3.2 \%$ and $3.6 \%$ proteases on average. In contrast, with only $2.3 \%$ and $2.8 \%$ protease encoding genes on average, plant parasites and saprophytes contained less (Table 2, Additional File 6). However, these average percentages are arguably not that different across fungal parasites and, again, considerable variation within host groups exists. As such, merely counting the number of proteases also does not seem to be as informative as previously suggested. Instead, taking a closer look at the types of proteases that are encoded in the genome might be more revealing. Other studies have linked aspartyl, serine, and subtilisin-like proteases to mycoparasitism (Viterbo et al. 2004). Indeed, the P. oviedoensis genome contained each of these protease types and even had an overrepresentation of serine-type proteases among the genes that were found to be unique to this species. However, N. ophiocordycipitis did not have any aspartyl or subtilisin-like proteases. In addition, previous studies have shown an upregulation of trypsin-like proteases during mycoparasite interaction with host mycelia (Troian et al. 2014). However, the $P$. oviedoensis genome did not contain any trypsin-like proteases while the $N$. ophiocordycipitis genome did. As such, also by going into greater detail, protease annotations do not appear to provide genome signatures that are consistent enough across mycoparasites to aid in their distinguishing from other parasitic lifestyles. 
Table 2

Annotated proteases and chitinases. The percentage of genes in each genome, annotated to encode for proteases is indicated in this table, as well as the number of genes encoding for glycoside hydrolase family 18 chitinases.

\begin{tabular}{|c|c|c|}
\hline Species & \% Protease Encoding Genes & Number of Chitinase Encoding Genes \\
\hline \multicolumn{3}{|l|}{ New Mycoparasite Species } \\
\hline Polycephalomyces oviedoensis & $3.16 \%$ & 13 \\
\hline Niveomyces ophiocordycipitis & $3.57 \%$ & 16 \\
\hline \multicolumn{3}{|l|}{ Mycoparasites } \\
\hline Tolypocladium ophioglossoides & $2.95 \%$ & 26 \\
\hline Trichoderma virens & $3.21 \%$ & 32 \\
\hline Trichoderma atroviride & $3.28 \%$ & 18 \\
\hline Trichoderma reesei & $3.22 \%$ & 27 \\
\hline Escovopsis weberi & $3.03 \%$ & 16 \\
\hline Clonostachys rosea & $3.57 \%$ & 15 \\
\hline \multicolumn{3}{|l|}{ Saprophytes } \\
\hline Ophiostoma piceae & $3.03 \%$ & 14 \\
\hline Stachybotrys chlorohalonata & $2.94 \%$ & 19 \\
\hline Neurospora crassa & $2.51 \%$ & 13 \\
\hline \multicolumn{3}{|l|}{ Animal Parasites } \\
\hline Ophiocordyceps camponoti-floridani & $3.67 \%$ & 11 \\
\hline Metarhizium robertsii & $3.75 \%$ & 17 \\
\hline Beauveria bassiana & $3.71 \%$ & 29 \\
\hline Pochonia chlamydosporia & $3.27 \%$ & 21 \\
\hline \multicolumn{3}{|l|}{ Plant Parasites } \\
\hline Fusarium oxysporum & $1.74 \%$ & 23 \\
\hline Claviceps purpurea & $2.87 \%$ & 9 \\
\hline
\end{tabular}

Chitin provides structural stability for fungal cell walls. As such, chitinases are important to fungi because of their involvement in several primary processes, including external chitin decomposition, cell wall degradation, and remodeling (Hartl et al. 2012). However, chitinases are thought to be especially important to mycoparasites and insect parasites that would need to break down the chitin in the cell walls or exoskeletons of their hosts to enter them (Hartl et al. 2012). The most prominent family of chitinases in fungi is the Glycoside Hydrolase Family 18 (Hartl et al. 2012). Previous studies have proposed that a high number of these $\mathrm{GH} 18$ chitinases in the genome is a characteristic of mycoparasitism (Kubicek et al. 2011; Karlsson et al. 2015). As such, we asked if our new hyperparasite genomes indeed harbor a high number of GH18 chitinases and how this number compares to the numbers found in the other genomes in our study. For $P$. oviedoensis, we found 13 genes within the $\mathrm{GH} 18$ family and $85 \%$ of them had orthologs 
in all other compared species while $N$. ophiocordycipitis had $16 \mathrm{GH} 18$ genes of which half had orthologs in all other compared species (Table 2, Supplementary File 7). For both hyperparasites, the number of chitinases was lower than the average numbers for mycoparasites (i.e., $22 \mathrm{GH} 18$ genes) and animal parasites (i.e., $19.5 \mathrm{GH} 18$ genes). Instead, their numbers were more in line with the average numbers found for plant parasites and saprophytes (i.e., 16 and 13.5 $\mathrm{GH} 18$ chitinases, respectively). Again, the numbers of chitinases within each lifestyle group were highly variable across the genomes included for each group. The previous study that highlighted the importance of GH18 chitinases to mycoparasitism did specifically include other fungal lifestyles into their comparisons. However, their chosen representative species did not belong to the order Hypocreales (Karlsson et al. 2017). As such, a large number of GH18 chitinases is perhaps more characteristic of Hypocreales as a whole than mycoparasites specifically.

\section{Discussion}

Although fungal hyperparasitism is a world-wide phenomenon that is often recognized by mycologists and disease ecologists, very few mycoparasitic species of entomopathogenic fungi have been formally described so far. Several hyperparasites of Ophiocordyceps species have been formally described so far (Wang et al. 2015b, a; Zhong et al. 2016; Xiao et al. 2018). However, hyperparasites associated with behavior-manipulating Ophiocordyceps have only been noted in the field as an ecological aspect of those species (Andersen and Hughes 2012; Araújo et al. 2020). In this study, we present one new genus (Niveomyces) and two new fungal species, P. oviedoensis and $N$. ophiocordycipitis, that infect behavior manipulating Ophiocordyceps. We complemented our study with a natural history survey that aimed to determine the timing of the new species' emergence and potential effects on their fungal host in nature. This is in contrast with previously published reports that merely used the presence of fungal growth on Ophiocordyceps to classify a species as a hyperparasite. Additionally, we sequenced the genomes of the two new hyperparasite species proposed herein and compared them to the genomes of other species in the order Hypocreales. The aim of our comparative genomics efforts was to determine if these genomes contain features that would further confirm a mycoparasitic lifestyle. However, the two new fungal hyperparasite species reside in parts of the fungal phylogenetic tree for which presently only very limited data is available, and the genomes of closely related species have not been sequenced yet. The fact that the genome for $P$. oviedoensis is the first one sequenced among the 24 species of Polycephalomyces senso (Crous et al. 2004; Kepler et al. 2013) and that we had to erect a new genus to describe $N$. ophiocordycipitis highlights this lack of knowledge about these fungi. These data limitations complicated our comparative genomics efforts and emphasizes the need for the sequencing of additional genomes and conducting of future studies on hypocrealean hyperparasites.

Both of the hyperparasites that we discovered on O.camponoti-floridani in Central Florida are delineated by our comprehensive phylogeny as new species in the families Cordycipitaceae and Ophiocordycipitaceae of the order Hypocreales. One of the species, P. oviedoensis, placed confidently as a new species within the genus Polycephalomyces, as the sister to a clade that is strictly associated with insect parasites. However, many other species within Polycephalomyces exhibit mycoparasite lifestyles (Fig. 1). Moreover, reports on P. yunnanensis, on $O$. nutans and its stinkbug host (Wang et al. 2015b) and $P$. lianzhouensis coinfecting the larva host of $O$. crinalis (Wang et al. 2015a) suggest that some Polychephalomyces species might be able to act both as mycoparasites and entomopathogens. However, more detailed observations on a bigger range of specimens for both Polycephalomyces species would be needed to determine if this is actually the case. All previously described hyperparasites of Ophiocordyceps have been placed within Polycephalomyces (Wang et al. 2015b, a; Zhong et al. 2016; Xiao et al. 2018). This makes it difficult to determine the origin of $P$. oviedoensis mycoparasitism using the present data. However, the other new hyperparasite species resides within a part of the Hypocreales tree that, thus far, largely contains insect parasites. This suggests that its mycoparasitism might have evolved from a previous animal parasitic

Page $16 / 30$ 
relationship (Fig. 1). Nevertheless, important species data is clearly missing for this part of the phylogeny as demonstrated by the new genus we had to invoke for our hyperparasite species (Fig. 1). Despite lower bootstrap value supporting Niveomyces we did feel confident about erecting a new genus for the new hyperparasite species since the Blackwellomyces species in its sister clade are morphologically vastly different. Assessing the macromorphology, Niveomyces exhibits an asexual morph with snow white mycelium that produces multiple spiky synnemata on a subiculum, while Blackwellomyces in nature often produces only the sexual morph that is vivid orange to red. As for its micromorphology, Niveomyces exhibit a hymenium-like layer of conidiogenous cells with a unique crown of denticles on the apex (each producing a single conidia). In contrast, Blackwellomyces produces commonly two types of asexual morphs concomitantly; Marianneae, Evlachovaea and Acremonium-like, which usually produces clusters or chains of sticky conidia that remain attached to the conidiogenous cells (Mongkolsamrit et al. 2020). Both the morphological and lifestyle differences between Niveomyces and Blackwellomyces thus support our phylogenetic data to assign this species both a new species and a new genus name.

While little research has been done on the effects of fungal hyperparasites on their hosts, Polycephalomyces $s p$. GIMCC 3.570 has been found to shorten the lifespan and significantly reduce the amount of ascospores produced by O. sinensis (Zhong et al. 2016). Moreover, undescribed hyperparasites of 0 . camponoti-rufipedis have been found to 'castrate' their hosts and prevent transmission (Andersen et al. 2012). Therefore, it is parsimonious to state that both $N$. ophiocordycipitis and $P$. oviedoensis negatively affect $O$. camponoti-floridani transmission. Indeed, the majority of observed $P$. oviedoensis-infected samples did not grow an ascoma after hyperparasite infection, suggesting that this hyperparasite interferes with $O$. camponoti-floridani transmission. In addition, $N$. ophiocordycipitis seems to negatively impact $O$. camponoti-floridani transmission by largely preventing the growth of a new ascoma or by completely covering an existing stalk and ascoma before consuming it, which prevents the release of spores. Moreover, $O$. camponoti-floridani stalks appeared to have died after infection by either hyperparasite species. This suggests that $N$. ophiocordycipitis and $P$. oviedoensis also shorten the lifespan of their host and prevent the production and transmission of spores beyond the timespan of our field study. As such, both hyperparasites affect the disease ecology of Ophiocordyceps-ant infections. More studies are needed to confirm the extent of the impacts of $P$. oviedoensis and $N$. ophiocordycipitis on $O$. camponoti-floridani transmission.

The fungi that grow on Ophiocordyceps have always been considered hyperparasitic mycoparasites. However, one could perhaps argue that they could also be growing saprophytically on dead insect or fungal tissue or act as entomopathogens that co-infect the insect host of Ophiocordyceps. The latter is especially enticing considering the phylogenetic placement of both species among species of entomopathogenic fungi. However, based on their morphology, which indicates that both P. oviedoensis and $N$. ophiocordycipitis only grow on top of 0 . camponotifloridani tissue, it is more likely that they are indeed mycoparasites. Our field surveys support this conclusion. Although in some cases $P$. oviedoensis and $N$. ophiocordycipitis growth was observed less than a week after a new $O$. camponoti-floridani-infected ant cadaver was found, they also appeared up to nine months after initial Ophiocordyceps infection. Moreover, 0 . camponoti-floridani mycelium begins to emerge from a fresh cadaver within one to two days after its death, providing already sufficient tissue for the mycoparasite to inhabit. This seemingly rules out the possibility of either species being an entomopathogen that coinfects the ant. Moreover, already within the first hours after an ant's manipulation and following death, Ophiocordyceps completely colonizes the ant's body, consuming all ant tissue besides the cuticle to gain the energy needed to grow a stalk and fruiting body (de Bekker et al., 2015). This makes it unlikely that either of the two species that we describe here would be able to saprophytically consume the insect cadaver. Moreover, the appearance of both presumed hyperparasite species in the first few weeks after death of the ant host, when 0 . camponoti-floridani is fresh and actively growing, seems to rule out the possibility of either species being an opportunistic saprophyte that merely feeds off of dead 0 . camponoti-floridani tissue. In 
fact, even months after ant manipulation and death have taken place, Ophiocordyceps is often found to be alive and is still able to produce stalks with ascoma. Taken together, our morphological and natural history data confirm previous assumptions that fungi found in association with Ophiocordyceps species, that emerged from their previously infected insect hosts, are indeed mycoparasitic hyperparasites.

In addition to performing a species description, phylogeny and natural history study, we sequenced the genomes of $P$. oviedoensis and $\mathrm{N}$. ophiocordycipitis and compared them to available genomes of the most closely related fungi we could find that spanned a diversity of lifestyles. In doing so, we aimed to detect signatures of their mycoparasitic lifestyle. Nevertheless, while our analyses all largely pointed towards a parasitic lifestyle for both species, no clear distinction between mycoparasitism or animal parasitism could be made. This might reflect an animal parasitic origin for these mycoparasites, as our phylogeny suggests. However, this result could also be an indication that a more meaningful and conclusive analysis would have been achieved if we would have been able to compare the genomes to those of species that are more closely related. We were especially limited by the number of available mycoparasite genomes within the order Hypocreales beyond the genus Trichoderma. This argues for the sequencing and investigation of more mycoparasites while better knowledge of their infection biology might hold keys to treating animal mycoses and the biological control of devastating crop diseases.

Indeed, our orthologous clustering analysis did not directly reflect a mycoparasitic lifestyle as both $P$. oviedoensis and $N$. ophiocordycipitis shared more unique orthogroups with the genomes of the fungal animal parasites that we included in our study than with the other mycoparasites. Yet, the unique orthogroups that were shared with plant parasites and saprophytes were clearly lower. When we investigated shared orthogroups for the presence of gene functions involved in infection biology and antagonistic interactions with other microbes, we found that genes with such functions were shared with both mycoparasites and animal parasites. In fact, we found that SSPs were overrepresented among the genes that were found to be unique to either new hyperparasite species, and those that were uniquely shared with animal parasites and both animal and mycoparasites. Small secreted proteins are often bioactive compounds that have a strong link to self-protection and host association (Kim et al. 2016). As such, both new hyperparasite species appear to have infection strategies that are unique to either species, as well as shared with other Hypocrealean parasites. Quite some of these SSPs were annotated to be toxins or antimicrobials, which further indicates that they likely play a role in the infection of 0 . camponoti-floridani. In addition, the high number of uncharacterized SSPs highlights the potential for new bioactive compound discovery in these species that could lead to the development of new applicable antifungals.

Our further comparative genomics analyses followed the lead of previous studies that have singled out high numbers of secondary metabolite clusters, proteases, and GH18 chitinases as genome signatures of a mycoparasitic lifestyle. However, these studies either did not include other lifestyles beyond mycoparasitism in their comparisons (Druzhinina et al. 2012; Karlsson et al. 2015), or chose representative species for other lifestyles outside of the order Hypocreales (Karlsson et al. 2017). We found differences in numbers of secondary metabolites, proteases, and chitinases that were not consistent or not large enough between lifestyle groups to be conclusive. As such, our results show that the functional gene categories discussed in earlier studies are not necessarily informative when conducting cross-lifestyle comparisons within the order Hypocreales. Instead, some of the suggested genome signatures may be merely characteristic of Hypocreales as a whole. Nevertheless, the numbers of functional annotations that we obtained for the secondary metabolite clusters, proteases and chitinases in the P. oviedoensis and $N$. ophiocordycipitis genomes, did generally place these species among the averages for mycoparasites and animal parasites, which is in line with our orthologous clustering results. As such, our genome comparisons could be indicating that fungal parasites that infect animal and fungal hosts largely evolved similar strategies, while plant parasites use other strategies. Alternatively, this could be another suggestion that the mycoparasitic species that infect 0 . camponoti-floridani

Page 18/30 
evolved from entomopathogens. Indeed, both hyperparasites have at least one gene that is annotated to encode for an enterotoxin. Beyond fungal animal parasites (which contain many enterotoxins) most fungal genomes do not contain a single PFAM annotation for this function (de Bekker et al. 2017). In line with this, none of the other mycoparasitic fungi in our study contain any putative enterotoxins in their genomes. As such, the remaining enterotoxin genes could indicate an earlier entomopathogenic ancestry for both P. oviedoensis and $N$. ophiocordycipitis.

\section{Conclusion}

To understand the complex dynamics of inter-species interactions more thoroughly, there is a need to incorporate tritrophic levels. Nevertheless, the natural enemies of parasites in parasite-host interactions are still vastly understudied. Our study contributes systematics, natural history, and genomic data to the current very limited knowledge of fungal hyperparasites of entomopathogenic fungi to shed a light on hyperparasitism and advocate for more in-depth investigations of fungal species that infect other fungi. We formally described and sequenced the genomes of two new hyperparasite species that infect the ant behavior manipulating parasite 0 . camponoti-floridani: Polycephalomyces oviedoensis and Niveomyces ophiocordycipitis. Our need to describe a new genus within the family Cordycipitaceae for one of the new species highlights the current limited data for this part of the Hypocreales phylogenetic tree. Moreover, our study adds considerable data to the very limited number of available mycoparasite genomes by providing two new genomes, of which one is the first genome in the genus Polycephalomyces. Our natural history data suggests that both species described in this study impact the transmission of the behaviormanipulating ant parasite 0 . camponoti-floridani, warranting further detailed studies into this tri-trophic fungusfungus-ant system. More detailed integrative studies into the infection biology of these and other mycoparasitic fungi, from the molecular to the ecological level, will result in the leveraging of new strategies to battle mycoses. Such discoveries are especially timely with fungal disease being on the rise.

\section{Declarations}

Ethics Approval and Consent to Participate

Not applicable

Adherence to National and International Regulations

Not applicable

Consent for Publication

Not applicable

Availability of Data and Material

The annotated genomes are deposited in GenBank: accession numbers JADHZA000000000 (Polycephalomyces oviedoensis) and JAFEME000000000 (Niveomyces ophiocordycipitis). The genome assembly, gene predictions and functional annotations can also be analyzed interactively at http://fungalgenomics.science.uu.nl. All other data has been included as additional files to this manuscript.

Competing Interests

Page 19/30 
The authors declare that they have no competing interests.

Funding

The work presented in this manuscript has been supported by startup funds from the University of Central Florida, made available to $\mathrm{CdB}$.

Author's Contributions

This study was conceived by BML and CdB who both also collected the samples used in this study and have written the manuscript. All other authors contributed to editing the manuscript. BML isolated the DNA, assembled the genomes, performed the functional annotations and comparative genomics analyses, and analyzed the field data. JPMA performed the phylogenetic analysis and the taxonomy. SRL collected the field data. RAO annotated the genomes and $A B$ performed the library preparations and genome sequencing.

Acknowledgements

We would like to thank Tatiana Sanjuan for early conversations about fungal hyperparasites of ant infecting Ophiocordyceps species and Davide Dal Pos for assisting with the macrophotography of our samples.

\section{References}

Andersen G, Yusah, et al (2009) The Life of a Dead Ant: The Expression of an Adaptive Extended Phenotype. Am Nat 174:. https://doi.org/10.2307/40306069

Andersen SB, Ferrari M, Evans HC et al (2012) Disease Dynamics in a Specialized Parasite of Ant Societies. PLoS One 7:. https://doi.org/10.1371/journal.pone.0036352

Andersen SB, Hughes DA (2012) Host specificity of parasite manipulation. Commun Integr Biol 5:. https://doi.org/10.4161/cib.18712

Andrews S (2010) FastQC: A Quality Control Tool for High Throughput Sequence Data

Araújo JPM, Evans HC, Fernandes IO et al (2020) Zombie-ant fungi cross continents: II. Myrmecophilous hymenostilboid species and a novel zombie lineage. Mycologia 112:.

https://doi.org/10.1080/00275514.2020.1822093

Araújo JPM, Evans HC, Kepler R, Hughes DP (2018) Zombie-ant fungi across continents: 15 new species and new combinations within Ophiocordyceps. I. Myrmecophilous hirsutelloid species. Stud Mycol 90:.

https://doi.org/10.1016/j.simyco.2017.12.002

Araújo JPM, Hughes DP (2016) Diversity of Entomopathogenic Fungi. Which Groups Conquered the Insect Body? Adv Genet 94:1-39. https://doi.org/10.1016/bs.adgen.2016.01.001

Araújo JPM, Hughes DP (2017) The fungal spore: myrmecophilous Ophiocordyceps as a case study. J Dighton, JM White (Eds), Fungal Community Its Organ Role Ecosyst CRC Press USA

Araújo JPM, Hughes DP (2019) Zombie-Ant Fungi Emerged from Non-manipulating, Beetle-Infecting Ancestors. Curr Biol 29:3735-3738. . https://doi.org/10.1016/j.cub.2019.09.004 
Asai S, Ayukawa Y, Gan P et al (2019) High-Quality Draft Genome Sequence of Fusarium oxysporum $f$. sp. cubense Strain 160527, a Causal Agent of Panama Disease. Microbiol Resour Announc 8:.

https://doi.org/10.1128/MRA.00654-19

Baker SE, Schackwitz W, Lipzen A et al (2015) Draft Genome Sequence of Neurospora crassa Strain FGSC 73. Genome Announc 3:. https://doi.org/10.1128/genomeA.00074-15

Bankevich A, Nurk S, Antipov D et al (2012) SPAdes: A New Genome Assembly Algorithm and Its Applications to Single-Cell Sequencing. J Comput Biol 19:. https://doi.org/10.1089/cmb.2012.0021

Blin K, Shaw S, Steinke K et al (2019) antiSMASH 5.0: updates to the secondary metabolite genome mining pipeline. Nucleic Acids Res 47:. https://doi.org/10.1093/nar/gkz310

Brakhage AA (2013) Regulation of fungal secondary metabolism. Nat Rev Microbiol 11:. https://doi.org/10.1038/nrmicro2916

Citores L, Iglesias R, Ragucci S et al (2018) Antifungal Activity of a-Sarcin against Penicillium digitatum: Proposal of a New Role for Fungal Ribotoxins. ACS Chem Biol 13:. https://doi.org/10.1021/acschembio.8b00410

Crous PW, Gams W, Stalpers JA et al (2004) MycoBank: An online initiative to launch mycology into the 21st century. Stud Mycol 50:19-22

de Bekker C, Ohm RA, Evans HC et al (2017) Ant-infecting Ophiocordyceps genomes reveal a high diversity of potential behavioral manipulation genes and a possible major role for enterotoxins. Sci Rep 7:.

https://doi.org/10.1038/s41598-017-12863-w

de Bekker C, Ohm RA, Loreto RG et al (2015) Gene expression during zombie ant biting behavior reflects the complexity underlying fungal parasitic behavioral manipulation. BMC Genom 16:. https://doi.org/10.1186/s12864015-1812-x

de Man TJB, Stajich JE, Kubicek CP et al (2016) Small genome of the fungus Escovopsis weberi, a specialized disease agent of ant agriculture. Proc Natl Acad Sci 113:. https://doi.org/10.1073/pnas.1518501113

Druzhinina IS, Shelest E, Kubicek CP (2012) Novel traits of Trichoderma predicted through the analysis of its secretome. FEMS Microbiol Lett 337:. https://doi.org/10.1111/j.1574-6968.2012.02665.x

El-Gebali S, Mistry J, Bateman A et al (2019) The Pfam protein families database in 2019. Nucleic Acids Res 47:. https://doi.org/10.1093/nar/gky995

Emms DM, Kelly S (2019) OrthoFinder: phylogenetic orthology inference for comparative genomics. Genome Biol 20:. https://doi.org/10.1186/s13059-019-1832-y

Evans HC, Elliot SL, Hughes DP (2011) Hidden diversity behind the zombie-ant fungus ophiocordyceps unilateralis: Four new species described from carpenter ants in Minas Gerais, Brazil. PLoS One 6:1-9.

https://doi.org/10.1371/journal.pone.0017024

Feau N, Dutech C, Brusini J, et al (2014) Multiple introductions and recombination in $<s c p>C</ s c p>$ ryphonectria hypovirus 1: perspective for a sustainable biological control of chestnut blight. Evol Appl 7:.

https://doi.org/10.1111/eva.12157

Page $21 / 30$ 
Frainer A, McKie BG, Amundsen P-A et al (2018) Parasitism and the Biodiversity-Functioning Relationship. Trends Ecol Evol 33:. https://doi.org/10.1016/j.tree.2018.01.011

Gleason FH, Lilje O, Marano AV et al (2014) Ecological functions of zoosporic hyperparasites. Front Microbiol 5:. https://doi.org/10.3389/fmicb.2014.00244

Gotz S, Garcia-Gomez JM, Terol J et al (2008) High-throughput functional annotation and data mining with the Blast2GO suite. Nucleic Acids Res 36:. https://doi.org/10.1093/nar/gkn176

Gurevich A, Saveliev V, Vyahhi N, Tesler G (2013) QUAST: quality assessment tool for genome assemblies. Bioinformatics 29:. https://doi.org/10.1093/bioinformatics/btt086

Haridas S, Wang Y, Lim L et al (2013) The genome and transcriptome of the pine saprophyte Ophiostoma piceae, and a comparison with the bark beetle-associated pine pathogen Grosmannia clavigera. BMC Genom 14:.

https://doi.org/10.1186/1471-2164-14-373

Hartl L, Zach S, Seidl-Seiboth V (2012) Fungal chitinases: Diversity, mechanistic properties and biotechnological potential. Appl Microbiol Biotechnol 93:533-543. https://doi.org/10.1007/s00253-011-3723-3

Hu X, Xiao G, Zheng P et al (2014) Trajectory and genomic determinants of fungal-pathogen speciation and host adaptation. Proc Natl Acad Sci 111:. https://doi.org/10.1073/pnas.1412662111

Hughes DP, Andersen SB, Hywel-Jones NL et al (2011) Behavioral mechanisms and morphological symptoms of zombie ants dying from fungal infection. BMC Ecol 11:. https://doi.org/10.1186/1472-6785-11-13

Jia S-L, Ma Y, Chi Z et al (2019) Genome sequencing of a yeast-like fungal strain P6, a novel species of Aureobasidium spp.: insights into its taxonomy, evolution, and biotechnological potentials. Ann Microbiol 69:. https://doi.org/10.1007/s13213-019-01531-1

Karlsson M, Atanasova L, Funck Jensen D, Zeilinger S (2017) Necrotrophic Mycoparasites and Their Genomes. https://doi.org/10.1128/microbiolspec.FUNK

Karlsson M, Durling MB, Choi J et al (2015) Insights on the Evolution of Mycoparasitism from the Genome of Clonostachys rosea. Genome Biol Evol 7:. https://doi.org/10.1093/gbe/evu292

Katoh K, Standley DM (2013) MAFFT multiple sequence alignment software version 7: Improvements in performance and usability. Mol Biol Evol 30:772-780. https://doi.org/10.1093/molbev/mst010

Keegan KP, Glass EM, Meyer F (2016) MG-RAST, a Metagenomics Service. for Analysis of Microbial Community Structure and Function

Kepler R, Ban S, Nakagiri A et al (2013) The phylogenetic placement of hypocrealean insect pathogens in the genus Polycephalomyces: An application ofOne Fungus One Name. Fungal Biol 117:611-622.

https://doi.org/10.1016/j.funbio.2013.06.002

Kepler RM, Luangsa-Ard JJ, Hywel-Jones NL et al (2017) A phylogenetically-based nomenclature for Cordycipitaceae (Hypocreales). IMA Fungus 8:335-353. https://doi.org/10.5598/imafungus.2017.08.02.08

Kim K-T, Jeon J, Choi J et al (2016) Kingdom-Wide Analysis of Fungal Small Secreted Proteins (SSPs) Reveals their Potential Role in Host Association. Front Plant Sci 7:. https://doi.org/10.3389/fpls.2016.00186

Page 22/30 
Krogh A, Larsson B, von Heijne G, Sonnhammer EL (2001) Predicting transmembrane protein topology with a hidden markov model: application to complete genomes11Edited by F. Cohen. J Mol Biol 305:

https://doi.org/10.1006/jmbi.2000.4315

Kubicek CP, Herrera-Estrella A, Seidl-Seiboth V et al (2011) Comparative genome sequence analysis underscores mycoparasitism as the ancestral life style of Trichoderma. Genome Biol 12.. https://doi.org/10.1186/gb-2011-12-4-r40

Kulkarni RD, Kelkar HS, Dean RA (2003) An eight-cysteine-containing CFEM domain unique to a group of fungal membrane proteins. Trends Biochem Sci 28:. https://doi.org/10.1016/S0968-0004(03)00025-2

Labaude S, Rigaud T, Cézilly F (2015) Host manipulation in the face of environmental changes: Ecological consequences. Int J Parasitol Parasites Wildl 4:. https://doi.org/10.1016/j.ijppaw.2015.08.001

Larriba E, Jaime MDLA, Carbonell-Caballero $\mathrm{J}$ et al (2014) Sequencing and functional analysis of the genome of a nematode egg-parasitic fungus, Pochonia chlamydosporia. Fungal Genet Biol 65:

https://doi.org/10.1016/j.fgb.2014.02.002

Letunic I, Bork P (2019) Interactive Tree Of Life (iTOL) v4: recent updates and new developments. Nucleic Acids Res 47:. https://doi.org/10.1093/nar/gkz239

Martinez D, Berka RM, Henrissat B et al (2008) Genome sequencing and analysis of the biomass-degrading fungus Trichoderma reesei (syn. Hypocrea jecorina). Nat Biotechnol 26:. https://doi.org/10.1038/nbt1403

Miller MA, Pfeiffer W, Schwartz T (2012) The CIPRES science gateway: Enabling high-impact science for phylogenetics researchers with limited resources. ACM Int Conf Proceeding Ser.

https://doi.org/10.1145/2335755.2335836

Mongkolsamrit S, Noisripoom W, Tasanathai K et al (2020) Molecular phylogeny and morphology reveal cryptic species in Blackwellomyces and Cordyceps (Cordycipitaceae) from Thailand. Mycol Prog 19:.

https://doi.org/10.1007/s11557-020-01615-2

Monod M, Capoccia S, Léchenne B et al (2002) Secreted proteases from pathogenic fungi. Int J Med Microbiol 292:. https://doi.org/10.1078/1438-4221-00223

Moussaïf M, Jacques P, Schaarwächter P et al (1997) Cyclosporin C is the main antifungal compound produced by Acremonium luzulae. Appl Environ Microbiol 63:. https://doi.org/10.1128/AEM.63.5.1739-1743.1997

Nielsen H, Engelbrecht J, Brunak S, von Heijne G (1997) Identification of prokaryotic and eukaryotic signal peptides and prediction of their cleavage sites. Protein Eng Des Sel 10:. https://doi.org/10.1093/protein/10.1.1

Ola ARB, Debbab A, Aly AH et al (2014) Absolute configuration and antibiotic activity of neosartorin from the endophytic fungus Aspergillus fumigatiaffinis. Tetrahedron Lett 55:. https://doi.org/10.1016/j.tetlet.2013.12.070

Park M-J, Choi Y-J, Hong S-B, Shin H-D (2010) Genetic variability and mycohost association of Ampelomyces quisqualis isolates inferred from phylogenetic analyses of ITS rDNA and actin gene sequences. Fungal Biol 114:. https://doi.org/10.1016/j.funbio.2010.01.003

Parra G, Bradnam K, Korf I (2007) CEGMA: a pipeline to accurately annotate core genes in eukaryotic genomes. Bioinformatics 23:. https://doi.org/10.1093/bioinformatics/btm071 
Parratt SR, Laine A-L (2016) The role of hyperparasitism in microbial pathogen ecology and evolution. ISME J 10: https://doi.org/10.1038/ismej.2015.247

Pontoppidan M-B, Himaman W, Hywel-Jones NL et al (2009) Graveyards on the Move: The Spatio-Temporal Distribution of Dead Ophiocordyceps-Infected Ants. PLoS One 4:. https://doi.org/10.1371/journal.pone.0004835

Prados-Rosales RC, Roldán-Rodríguez R, Serena C et al (2012) A PR-1-like Protein of Fusarium oxysporum Functions in Virulence on Mammalian Hosts. J Biol Chem 287:. https://doi.org/10.1074/jbc.M112.364034

Prasad R, Rawal MK (2014) Efflux pump proteins in antifungal resistance. Front Pharmacol 5:. https://doi.org/10.3389/fphar.2014.00202

Preston DL, Mischler JA, Townsend AR, Johnson PTJ (2016) Disease Ecology Meets Ecosystem Science. Ecosystems 19:. https://doi.org/10.1007/s10021-016-9965-2

Quandt CA, Bushley KE, Spatafora JW (2015) The genome of the truffle-parasite Tolypocladium ophioglossoides and the evolution of antifungal peptaibiotics. BMC Genom 16:. https://doi.org/10.1186/s12864-015-1777-9

Rawlings ND, Barrett AJ, Thomas PD et al (2018) The MEROPS database of proteolytic enzymes, their substrates and inhibitors in 2017 and a comparison with peptidases in the PANTHER database. Nucleic Acids Res 46:.

https://doi.org/10.1093/nar/gkx1134

Schafhauser T, Kirchner N, Kulik A et al (2016) The cyclochlorotine mycotoxin is produced by the nonribosomal peptide synthetase CctN in Talaromyces islandicus (' Penicillium islandicum'). Environ Microbiol 18:.

https://doi.org/10.1111/1462-2920.13294

Semeiks J, Borek D, Otwinowski Z, Grishin NV (2014) Comparative genome sequencing reveals chemotype-specific gene clusters in the toxigenic black mold Stachybotrys. BMC Genom 15:. https://doi.org/10.1186/1471-2164-15-590

Seppey M, Manni M, Zdobnov EM (2019) BUSCO: Assessing Genome Assembly and Annotation Completeness

Stamatakis A (2014) RAxML version 8: a tool for phylogenetic analysis and post-analysis of large phylogenies. Bioinformatics 30:. https://doi.org/10.1093/bioinformatics/btu033

Stanke M, Morgenstern B (2005) AUGUSTUS: a web server for gene prediction in eukaryotes that allows user-defined constraints. Nucleic Acids Res 33:. https://doi.org/10.1093/nar/gki458

Steenwyk JL, Mead ME, Knowles SL et al (2020) Variation Among Biosynthetic Gene Clusters, Secondary Metabolite Profiles, and Cards of Virulence Across Aspergillus Species. Genetics 216:.

https://doi.org/10.1534/genetics.120.303549

Sung GH, Hywel-Jones NL, Sung JM et al (2007) Phylogenetic classification of Cordyceps and the clavicipitaceous fungi. Stud Mycol 57:5-59. https://doi.org/10.3114/sim.2007.57.01

Thompson JN (1999) The Evolution of Species Interactions. Science (80-) 284:.

https://doi.org/10.1126/science.284.5423.2116

Troian RF, Steindorff AS, Ramada MHS et al (2014) Mycoparasitism studies of Trichoderma harzianum against Sclerotinia sclerotiorum: evaluation of antagonism and expression of cell wall-degrading enzymes genes. Biotechnol Lett 36:. https://doi.org/10.1007/s10529-014-1583-5

Page 24/30 
Uehling J, Deveau A, Paoletti M (2017) Do fungi have an innate immune response? An NLR-based comparison to plant and animal immune systems. PLOS Pathog 13.. https://doi.org/10.1371/journal.ppat.1006578

Viggiano A, Salo O, Ali H et al (2017) Pathway for the Biosynthesis of the Pigment Chrysogine by Penicillium chrysogenum. Appl Environ Microbiol 84:. https://doi.org/10.1128/AEM.02246-17

Viterbo A, Harel M, Chet I (2004) Isolation of two aspartyl proteases from expressed during colonization of cucumber roots. FEMS Microbiol Lett 238:. https://doi.org/10.1016/j.femsle.2004.07.028

Wang Y-B, Yu H, Dai Y-D et al (2015a) Polycephalomyces agaricus, a new hyperparasite of Ophiocordyceps sp. infecting melolonthid larvae in southwestern China. Mycol Prog 14:. https://doi.org/10.1007/s11557-015-1090-7

Wang YB, Yu H, Dai YD et al (2015b) Polycephalomyces yunnanensis (Hypocreales), a new species of Polycephalomyces parasitizing Ophiocordyceps nutans and stink bugs (hemipteran adults). Phytotaxa 208:034-044. https://doi.org/10.11646/phytotaxa.208.1.3

Will I, Das B, Trinh T et al (2020) Genetic underpinnings of host manipulation by Ophiocordyceps as revealed by comparative transcriptomics. bioRxiv 10:2275-2296. https://doi.org/10.1101/2020.01.03.893917

Windsor DA (1998) Controversies in parasitology, Most of the species on Earth are parasites. Int J Parasitol 28:. https://doi.org/10.1016/S0020-7519(98)00153-2

Wingfield BD, Liu M, Nguyen HDT et al (2018) Nine draft genome sequences of Claviceps purpurea s.lat., including $C$. arundinis, C. humidiphila, and C. cf. spartinae, pseudomolecules for the pitch canker pathogen Fusarium circinatum, draft genome of Davidsoniella eucalypti, Grosmannia galeiformis, Quambalaria eucalypti, and Teratosphaeria destructans. IMA Fungus 9:. https://doi.org/10.5598/imafungus.2018.09.02.10

Wu J, Gao B, Zhu S (2014) The Fungal Defensin Family Enlarged. Pharmaceuticals 7:. https://doi.org/10.3390/ph7080866

Wu Z, Williams LJ, Danishefsky SJ (2000) A three-step entry to the aspirochlorine family of antifungal agents. Angew Chemie - Int Ed 39:3866-3868. https://doi.org/10.1002/1521-3773(20001103)39:21<3866::AID-ANIE3866>3.0.CO;2-E

Xiao G, Ying S-H, Zheng P et al (2012) Genomic perspectives on the evolution of fungal entomopathogenicity in Beauveria bassiana. Sci Rep 2:. https://doi.org/10.1038/srep00483

Xiao Y-P, Wen T-C, Hongsanan S et al (2018) Multigene phylogenetics of Polycephalomyces (Ophiocordycipitaceae, Hypocreales), with two new species from Thailand. Sci Rep 8:. https://doi.org/10.1038/s41598-018-36792-4

Zhang Y, Wei W, Fan J et al (2020) Aspergillus fumigatus mitochondrial acetyl coenzyme a acetyltransferase as an antifungal target. Appl Environ Microbiol 86:1-16. https://doi.org/10.1128/AEM.02986-19

Zhong X, Li S, Peng Q et al (2016) A Polycephalomyces hyperparasite of Ophiocordyceps sinensis leads to shortened duration of production and reduced numbers of host ascospores. Fungal Ecol 21:

https://doi.org/10.1016/j.funeco.2016.03.002

\section{Figures}




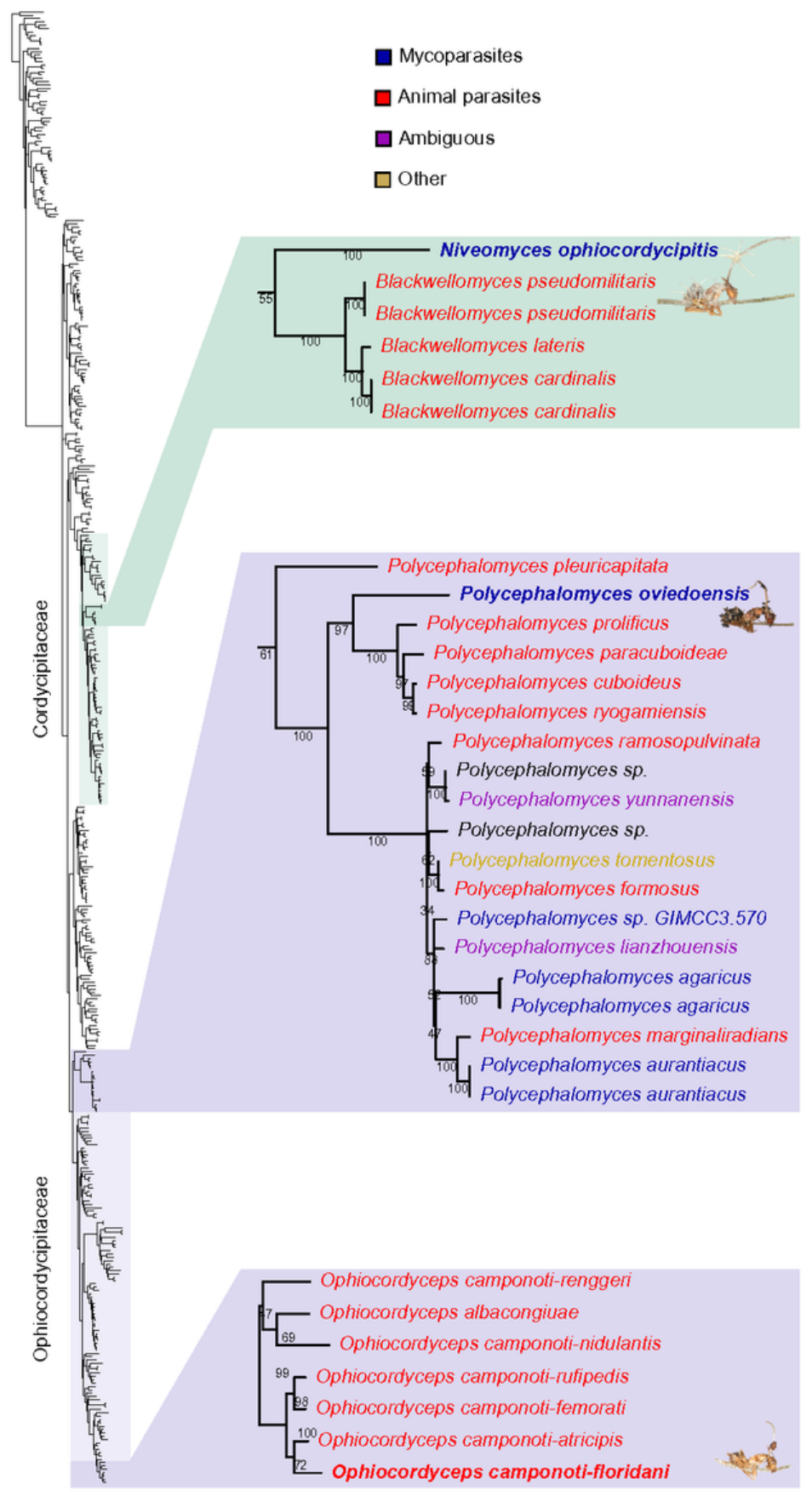

\section{Figure 1}

Phylogenetic placement of two new hyperparasitic fungal species of 0 . camponoti-floridani. A phylogenetic tree of the fungal order Hypocreales containing 445 representative species, including the two new species described in this study and their host $\mathrm{O}$. camponoti-floridani. The new species reside within the families Cordycipitaceae and Ophiocordycipitaceae, which have been highlighted. Additionally, the clades for the two new species P. oviedoensis and $\mathrm{N}$. ophiocordycipitis, as well as their host $\mathrm{O}$. camponoti-floridani, are shown. The colors indicate reported lifestyles for each of the fungal species that that we found to be closely related to the species that are the focus of this study. 


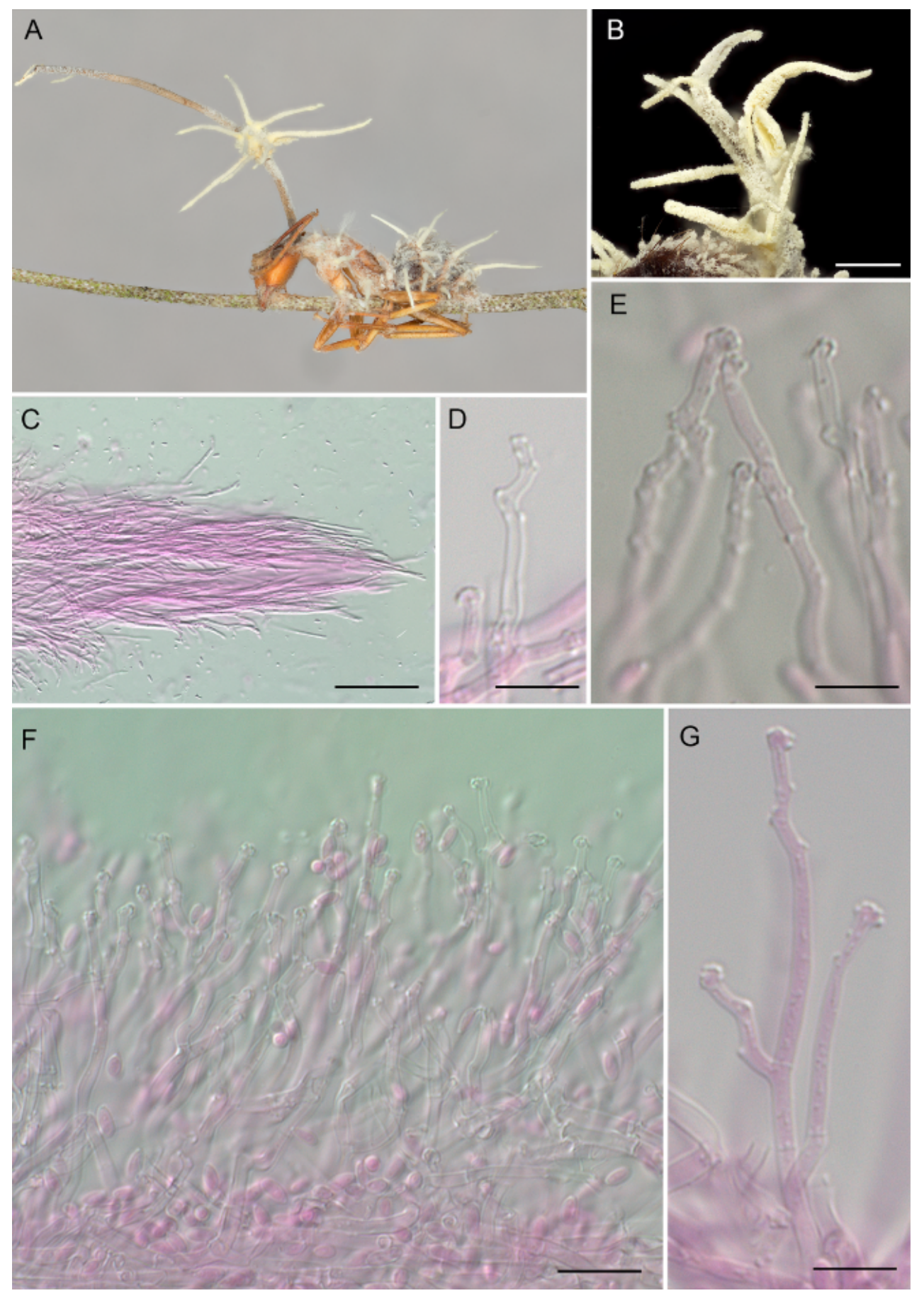

\section{Figure 2}

Niveomyces ophiocordycipitis (A) N. ophiocordycipitis growing on Ophiocordyceps camponoti-floridani that parasitizes Camponotus floridanus; (B) Close-up of synnemata;(C) Close-up of the apical part of synnema; (DE) Conidiogenous cells; (F) Hymenium-like layer of conidiogenous cells; (G) Close-up of conidiogenous cells with characteristic crown of denticles. Scale bars:(C) $200 \mu \mathrm{m}$;(D-F) $10 \mu \mathrm{m} ;(\mathrm{G}) 5 \mu \mathrm{m}$. 


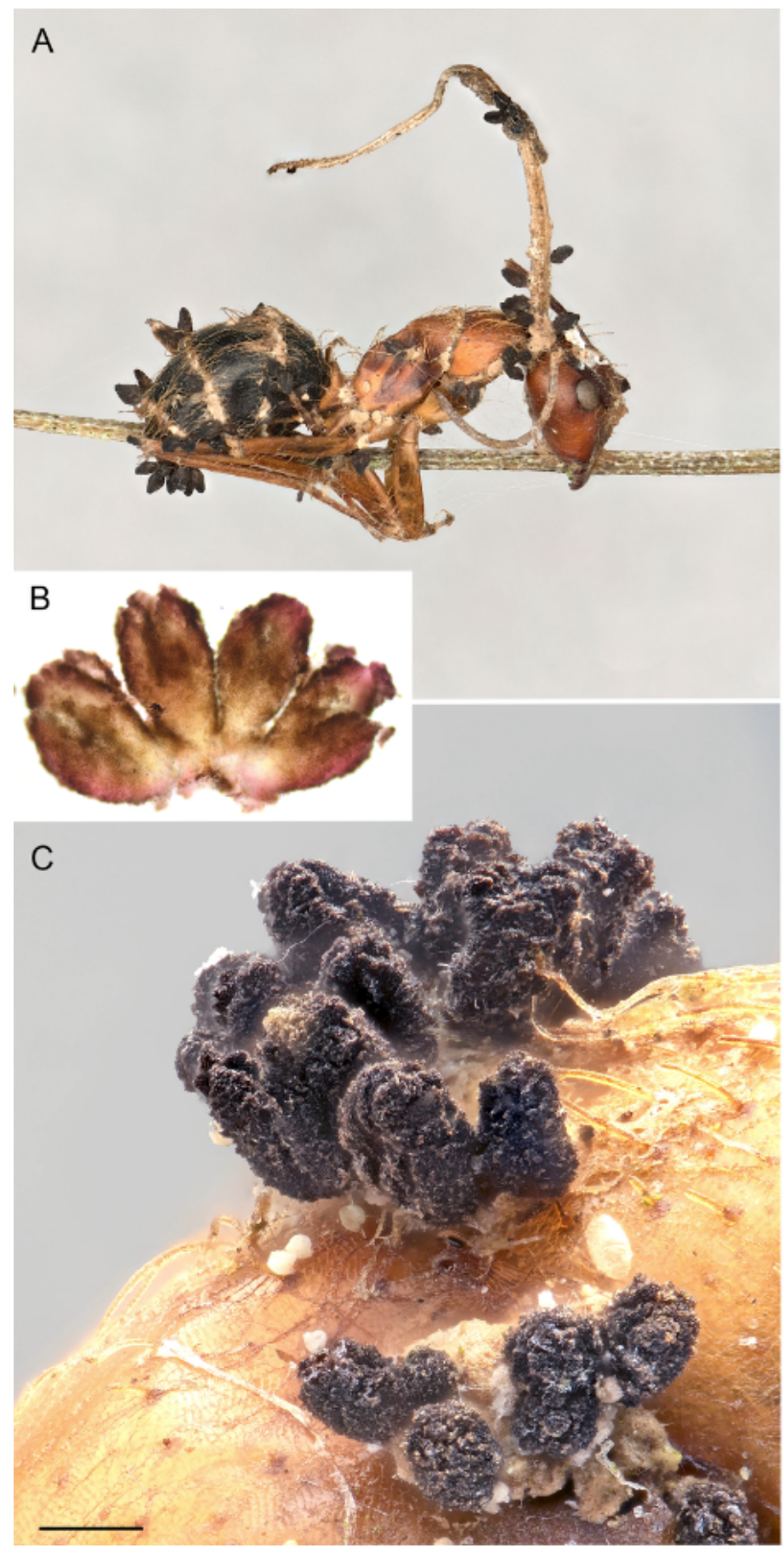

Figure 3

Polycephalomyces oviedoensis: (A) P. oviedoensis growing on Ophiocordyceps camponoti-floridani, which parasitizes Camponotus floridanus. (B) Perithecia under optical microscope; (C) Close-up of perithecia. 

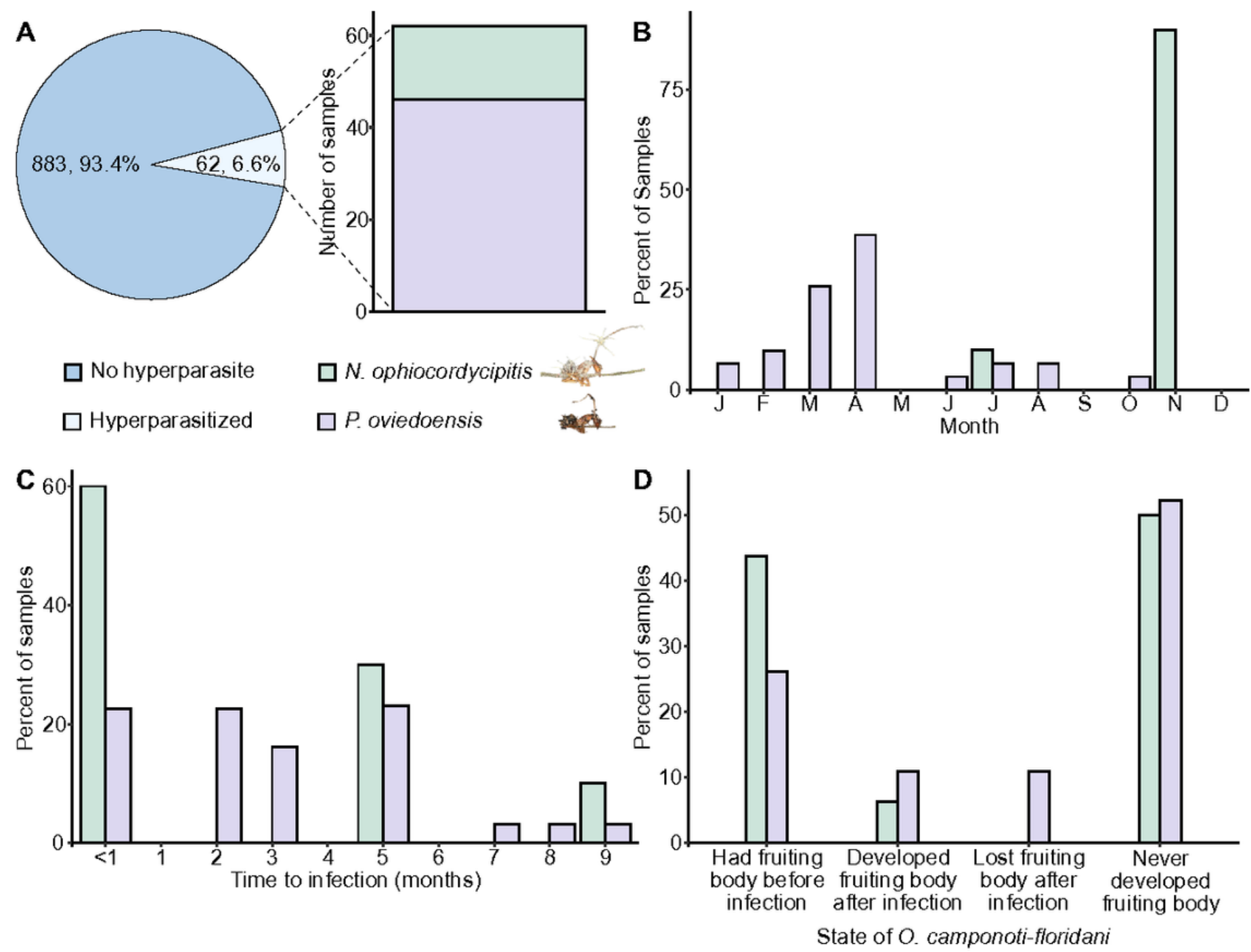

\section{Figure 4}

Hyperparasite infections of Ophiocordyceps camponoti-floridani in nature. (A) The number and percentage of 0. camponoti-floridani samples found infected with either P. oviedoensis or N. ophiocordycipitis. (B) The month in which a newly hyperparasitized cadaver was first noticed. (C) The time between the appearance of a newly Ophiocordycepsinfected carpenter ant and its first noticeable infection with either P. oviedoensis or N. ophiocordycipitis. (D) The state of 0 . camponoti-floridani after infection by a hyperparasite as measured by the presence or absence of a fruiting body. 
A

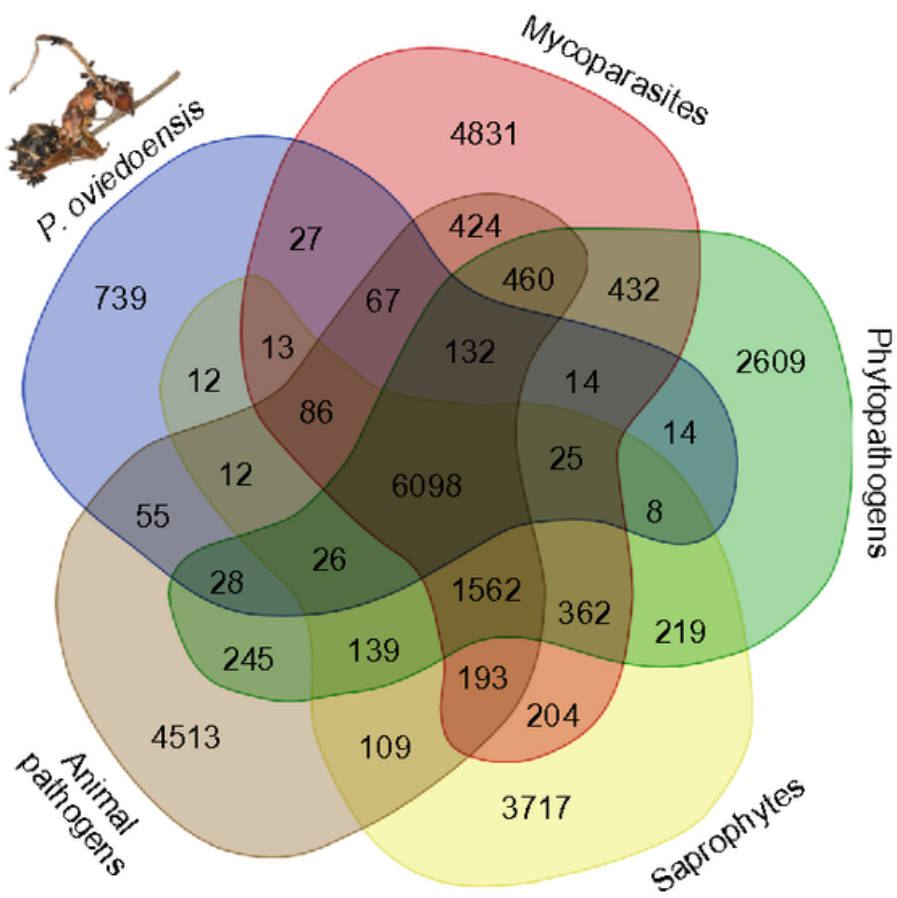

B

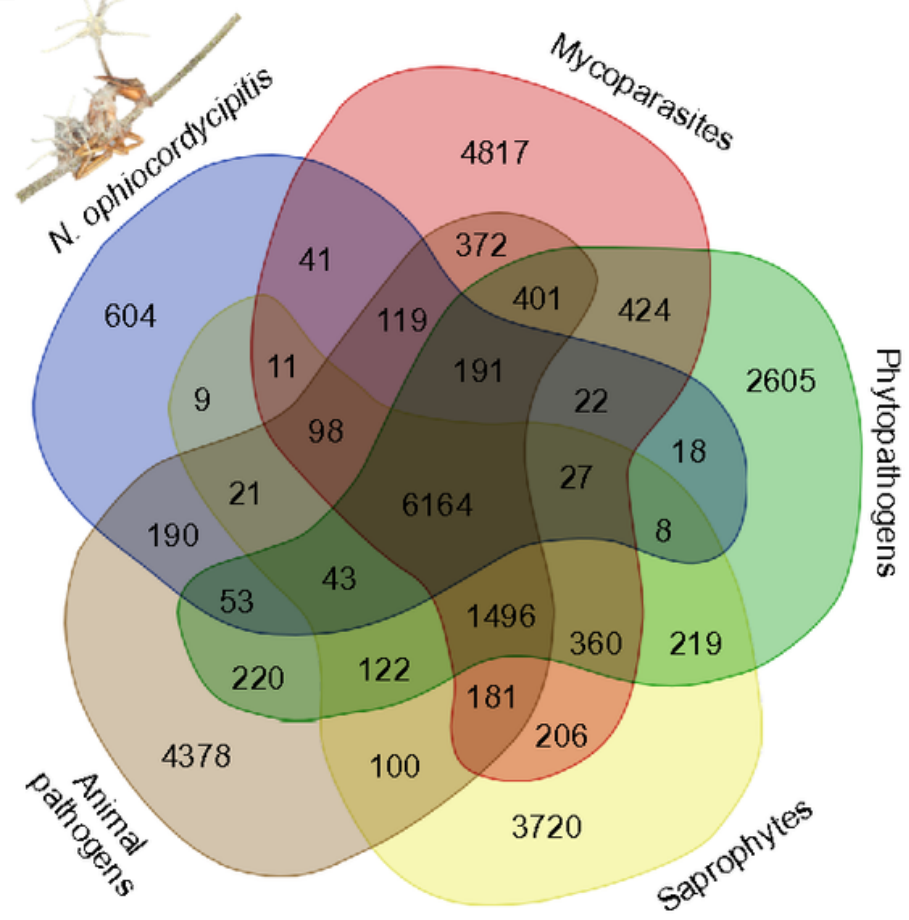

\section{Figure 5}

Orthologous clustering. Venn diagrams representing the shared orthogroups between the genomes of P. oviedoensis (A) and N. ophiocordycipitis (B) and the genomes of 15 other fungi that we grouped into different fungal lifestyles based on their ability to parasitize animal, fungal, or plant hosts, or decompose dead organic material.

\section{Supplementary Files}

This is a list of supplementary files associated with this preprint. Click to download.

- AdditionalFile1.xlsx

- AdditionalFile2.png

- AdditionalFile3.xlsx

- AdditionalFile4.xlsx

- AdditionalFile5.xlsx

- AdditionalFile6.xlsx

- AdditionalFile7.xlsx 